\title{
DnaK response to expression of protein mutants is dependent on translation rate and stability
}

\section{Author affiliation}

5 Signe Christensen, Department of Biochemistry and Structural Biology, Lund University,

6 Lund, Sweden

7 Sebastian Rämisch, Max Planck Unit for the Science of Pathogens, Berlin, Germany

8 Ingemar André, Department of Biochemistry and Structural Biology, Lund University, Lund,

9 Sweden

\section{Abstract}

13 Chaperones play a central part in the quality control system in cells by clearing misfolded and

14 aggregated proteins. The chaperone DnaK acts as a sensor for molecular stress by recognising

15 short hydrophobic stretches of misfolded proteins. As the level of unfolded protein is a function

16 of protein stability, we hypothesised that the level of DnaK response upon overexpression of

17 recombinant proteins would be correlated to stability. Using a set of mutants of the $\lambda$-repressor with varying thermal stabilities and a fluorescent reporter system, the effect of stability on

19 DnaK response and protein abundance was investigated. Our results demonstrate that the initial

20 DnaK response is largely dependent on protein synthesis rate but as the recombinantly expressed protein accumulates and homeostasis is approached the response correlates strongly abundance and DnaK response in more stable proteins. 


\section{Introduction}

27 The production of functional proteins typically requires folding into well-defined threedimensional structures. However, protein synthesis can also lead to accumulation of unfolded, misfolded, or aggregated protein due to their marginal stability. As aggregated and misfolded proteins can have toxic effects on the cell there is a substantial fitness cost associated with them. To reduce the harm caused by aberrant proteins all living organisms have developed a sophisticated protein quality control (PQC) network. The PQC is a collection of chaperones and proteases that assists with refolding of misfolded proteins, protects from irreversible aggregation by formation of inclusion bodies or, eventually, direct misfolded or aggregated proteins for degradation. Due to the ubiquitous nature of the PQC the involved proteins are required to be highly promiscuous. E.g., hsp70 (known as DnaK in E. coli) binds to hydrophobic stretches approximately five residues long, that are exposed upon misfolding [1].

These short hsp70/DnaK-binding motifs are present in most proteins and allow for recognition of unfolded or misfolded proteins, in a matter largely independent of sequence and structure.

Recognition of unfolded and misfolded proteins by DnaK is one of the major regulators of the transcription factor $\sigma^{32}$ and hence PQC network in E. coli, although $\sigma^{32}$ is also regulated by e.g. GroEL/S [2]. Under low stress conditions DnaK binds to $\sigma^{32}$ targeting it for degradation by the metallo-protease FtsH [3]. In response to stress, DnaK binds preferentially to misfolded protein instead of $\sigma^{32}$ allowing $\sigma^{32}$ to act as a transcription factor for expression of DnaK and other PQC proteins. Other proteins in the PQC network that are regulated by $\sigma^{32}$ includes chaperones involved in protein folding and disaggregation (ClpB, DnaJ, GroEL and GroES), cytosolic proteases that degrade proteins (ClpP, ClpX and Lon), as well as the membranebound $\mathrm{FtsH}$ involved in the regulation of $\sigma^{32}$ levels. 
50 Whereas it is well-established that misfolded proteins trigger a response from the PQC the molecular properties of the client proteins that impact its regulation is not fully understood.

52 One open question is whether this response is primarily determined by the concentration of unfolded protein, or if there is a sequence dependent component to the response beyond chaperone recognition sequences. The lower the stability of a protein translated within the cell, the less will be found in the folded native state. Consequently, if the PQC is primarily dependent on concentration of unfolded or misfolded protein a correlation between protein stability and PQC response should be expected. Whether such simple relationship exists is not clear, although upregulation of GroEL, c62.5 (hsp90 homolog) and DnaK [4, 5], has been observed upon introduction of mutations that destabilize wildtype sequences. A combination of computational models and in vitro studies performed by Sekhar et al. suggest that DnaK binds preferentially to unstable proteins at folding equilibrium, but to slow-folding proteins while folding[6]. As DnaK can interact both co-translationally with the unfolded nascent peptide chain and with misfolded protein [7] a similar process could occur in vivo. However, no in vivo studies of the correlation between folding rates and DnaK response has been presented.

Another central question that is not fully understood is how the chaperone response is regulated by protein production. Overexpression of proteins in E. coli results in increased levels of chaperones $[8,9]$, but it is not clear to what extent this response is controlled by the level of protein translation or simply the abundance of misfolded/unfolded proteins in the cytosol. There is a negative correlation between the level of expression of a protein in E. coli and its dependency on DnaK for folding [10]. Thus, proteins that are difficult to fold might express at lower levels. This indicates that the cell has limited capacity to process misfolded/unfolded proteins and perhaps also a limited range to regulate the chaperone response. Mutations does 
not only affect the folding stability and kinetics of proteins, but also the level of expression.

Therefore, to understand the regulation of the chaperone response also the protein expression and abundance must be considered.

The presence of misfolded/unfolded proteins carries a fitness cost to the cell [11-14]. By modifying the abundance of misfolded proteins, chaperones can modify the rate of protein sequence evolution [10, 15-21]. Hence, chaperones, like DnaK, can enable proteins to evolve faster. However, there is still an associated fitness cost as triggering the PQC response consumes resources; both directly by consumption of ATP and by occupying the synthesis machinery for production of chaperones and proteases forming the PQC network. In theory, the interaction of proteins with the PQC network can also impact which sequences are fixated during evolution. Protein variants triggering a large PQC response would be expected to have lower fitness and thus selected against. In light of this, information about how molecular variation impacts DnaK interaction and the PQC can provide valuable insight into the role of the PQC on protein evolution.

As summarised above the components of the PQC system and the regulation network are well described, but it has yet to be studied in detail how the biophysical properties of the client protein impact the chaperone response. Thus, in this study we investigated the effect of molecular variation on the PQC. More specifically, we focused on the following questions: (i) What is the relationship between protein stability and DnaK response? (ii) Is the DnaK response controlled by the level of protein translation, the amount of unfolded protein or both? (iii) How much does the PQC response vary with small variation in biophysical properties of protein variants? (iv) How much does the PQC response vary between individual cells? We used superfolder Green Fluorescent Proteins (sfGFP) expressed from the stress-inducible 
100 DnaK promoter to evaluate the effect on the PQC response upon introducing stability-altering

101 point mutations to the $\lambda$ repressor construct, N102LT. We found that induction of the DnaK

102 promoter is dependent on protein translation rates early after onset of recombinant protein

103 synthesis, but dependent on protein stability when homeostasis is reached. We further found

104 that protein stability has a significant impact on accumulation of protein in the cell due to

105 increased degradation of proteins with low stability and that the response varies substantially

106 between cells. The investigation also indicates how the fluorescence system can be used as a

107 high-throughput stability assay.

\section{Results}

\section{Monitoring protein accumulation and DnaK response with fluorescent}

\section{2 reporters}

113 To study how the biophysical properties of proteins impact the stress response in E. coli, we

114 constructed a reporter plasmid system to simultaneously monitor the abundance of 115 recombinantly expressed protein and chaperone response upon recombinant expression (Figure

116 1A). It contains two fluorescent reporters: (i) The red fluorescent protein tagRFP is fused to

117 the N-terminal of a protein of interest (POI), and expression of the POI-tagRFP fusion protein

118 is controlled by the IPTG inducible lac promoter. The fusion protein allows for monitoring the

119 abundance of recombinant POI in the cell. In addition, the folding state of the POI could impact

120 the folding of tagRFP and therefor acts as an additional stability reporter, as previously shown

121 for GFP-tagged proteins [22-24]. (ii) Expression of Superfolder Green Fluorescent Protein

122 (sfGFP) is controlled by the DnaK promoter to enable monitoring of the stress response in

123 individual cells. The PQC network consists of many different components, thus the GFP signal

124 gives only a partial picture of the overall state. But since DnaK is a central component in the 
response to folding stress we consider it a meaningful proxy for the PQC network. However, it is worth noticing that synthesis and degradation of PQC proteins are tightly regulated in the cell [25-27]. As the degradation of GFP is decoupled from the degradation of DnaK the GFP signal does not report on the current level of DnaK in the cell, but rather the accumulated amount of DnaK produced until that point. However, by evaluating the rate of GFP production

130 - which is correlated to the rate of DnaK synthesis - we can measure when the DnaK response

131 is triggered. The protein abundance reporter (tagRFP) and the DnaK reporter (sfGFP) are

132 located on the same plasmid. This enables normalization of the chaperone signal with protein 133 abundance so that cell-cell variation and variation in plasmid copy numbers can be accounted

134 for. It is important to note that even though a DnaK promoter is utilised for expression of GFP, 135 the endogenous DnaK promoter is still in place ensuring a functional PQC network in the cells.

136 As a model system for investigating the correlation between DnaK response and protein stability we use several variants of the $\lambda$ repressor construct, N102LT, with different thermodynamic stability. N102LT is a 128 amino acid long protein consisting of the N-terminal

139 domain of the $\lambda$ repressor extended by the C-terminal domain of the protein arc that protects

140 the fusion protein against proteolysis [29]. It has previously been used to study the heat shock

141 response in E. coli [5, 30-33]. To separate effects caused by changes in stability from effects

142 caused by site-specific structural factors it is beneficial to also evaluate variants with mutations

143 at the same position. In this study we therefor included seven variants with single point

144 mutations in four different positions: Q33, V36, M40 and L57 with in vitro melting temperatures from below $10{ }^{\circ} \mathrm{C}$ to $61{ }^{\circ} \mathrm{C}$ in addition to wild-type $\mathrm{N} 102 \mathrm{LT}\left(\mathrm{Tm}=55^{\circ} \mathrm{C}\right)$.

146 Position L57, M40 and V36 are part of the hydrophobic core, whereas Q33 is surface exposed

147 and is part of the cognate DNA recognition (Figure 1B). Mutations in position L57 and M40

148 resulted in four variants (L57C, L57G, L57P and M40A) with reduced thermostability 149 compared to the wt sequence, while V36I results in increased stability. Mutating the surface 
accessible Q33 to either serine (Q33S) or tyrosine (Q33Y) retains or increases thermostability, respectively.

153 Changes in the ability of the N102LT variants to bind DnaK could potentially impact the observed stress response. To check whether the introduced mutations cause significant changes to the DnaK binding properties we use the ChaperISM algorithm to predict DnaK binding properties for the eight variants [34] (S1). N102LT wt is predicted to have three DnaK binding sites. Introduction of the Q33Y mutation increases the DnaK binding propensity slightly around position 33, but without introducing a new DnaK binding site. Mutating position L57 reduces the DnaK binding propensity, but without disrupting a DnaK binding site.

Point mutations in N102LT results in differences in accumulation of protein,

163 To monitor changes in protein abundance and DnaK response the N102LT variants were expressed from the reporter plasmid in E. coli and grown in liquid media. Synthesis of the N102LT variants was induced with IPTG during exponential growth. Flow cytometry was employed to simultaneous monitor the abundance of protein (red fluorescence), DnaK transcription (green fluorescence), as well as morphological changes (forward and side scatter) on single-cell level. In addition to analysis by flow cytometry, cell growth was monitored by optic density at $600 \mathrm{~nm}\left(\mathrm{OD}_{600}\right)$ (Figure 2A, S2).

\section{Cell growth after induction of recombinant protein production is variant-dependent}

172 If variants trigger the PQC network to a varying degree, they might also have different impact

173 on cell fitness. To investigate this, we tracked cell density during protein expression of each 
175 of recombinant protein expression (Figure 2B), which is likely due to leaky expression.

176 Independent of which variant is expressed, the cells continue to grow for approximately two

177 hours after induction. After this point cells expressing the wt as well as Q33Y and V36I show

178 a drop in $\mathrm{OD}_{600}$ (Figure 2A, S2) indicating that cells expressing these variants are dying. After

179 induction of recombinant protein production there is greater variation in the growth rate of cells

180 expressing different variants, but with similar patterns as before induction. Neither the

181 preinduction nor postinduction growth rate correlates with protein stability $(2 \mathrm{~B}+\mathrm{C})$.

182

183 Accumulation of protein is stability-dependent

184 To monitor the abundance of protein in the cell we followed the development of red

185 fluorescence over time. The first $30 \mathrm{~min}$ after induction with IPTG the red fluorescence

186 intensity is close to constant followed by continuous increase throughout the four hours for

187 which protein production is monitored (Figure 3A+S3). Due to the long maturation time of

188 tagRFP ( $\mathrm{t}_{50} \sim 2 \mathrm{~min}$ [35]) the low red fluorescence levels the first 30 min does not necessarily

189 indicate a lack of protein synthesis. Throughout the entire experiment the red fluorescence

190 correlates well with variant melting temperature (Figure 3B), with a spearman rank correlation

191 of 0.83 after $120 \mathrm{~min}$. At that point, variants with melting temperatures higher than the wt have

192 red fluorescence intensities double to the variants with lower stabilities, with the exception of

193 one replicate of Q33Y (Figure 3C). Looking at different mutations at the same site we observe

194 that L57G and L57C have similar fluorescence, while the more stable L57P variant has an

195 increased value. This indicates that the decreased expression in the former variants is due to

196 stability rather than a consequence of mutating a particular position.

197 
Variation in protein accumulation is due to differences in rate of protein degradation rather than synthesis

200 The amount of accumulated protein is a consequence of the opposing effects of synthesis and

201 degradation. Variation in abundance between mutants can thus be explained by differences in

202 protein synthesis rate, protein degradation rate or both. We estimated the relative rate of red

203 fluorescence change (change in fluorescence intensity divided by the intensity) between 30 and 60 min ("early" rates), and between 60 and 90 min ("late" rates) after induction. Both late and early rates varies with variant (Figure 3D). Interestingly, for variants less stable than wt the late rates are significantly reduced compared to the early rates. Assuming degradation is minimal early after onset of protein synthesis, this indicates that eventually the rate of degradation surpasses the rate of synthesis for these variants. For the more stable variants (wt, Q33S, Q33Y and V36I) the relative rate is not reduced or only minimally reduced after $60 \mathrm{~min}$, which indicates that less protein is degraded in the cells harbouring these variants. The late rates correlate with thermostability with a spearman rank correlation of 0.7 (Figure 3D). Taken together, our results suggests that the difference in relative rate is most likely due to stabilitydependent degradation of variants rather than difference in the rate of translation.

218 Protein folding stress triggers the PQC network and we wished to investigate whether protein

219 translation alone is able to trigger a response and to what extent the biophysical properties, such as thermostability, of a protein impacts the stress response. 
222 Assuming that the degradation of GFP is independent of the variant expressed, the total green

223 fluorescence signal in Figure 4A and S4 reports on the total synthesis of GFP initiated at the

224 stress-controlled DnaK promoter. Therefore, it can serve as a proxy for the accumulated DnaK

225 response triggered by expression of the 8 variants. Immediately upon induction with IPTG an

226 increase in green fluorescence is observed (Figure 4A and S4) which shows that the sfGFP

227 signal emerge even before protein synthesis can be detected as an increase in red fluorescence.

228 However, this can be explained by a shorter maturation time for sfGFP than tagRFP (14 min

229 vs 42 minutes) [35]. Due to the much slower maturation time of tagRFP compared to sfGFP,

230 total fluorescent intensities are not synchronized like the molecular events in the cell. However,

231 by monitoring the rate of green and/or red fluorescence instead of total fluorescence we

232 eliminate the effect of different maturation times.

233

234 Protein translation alone triggers DnaK transcription

235 The initial rate (0 to $30 \mathrm{~min}$ after IPTG induction) of green fluorescence correlates strongly

236 with the initial rate of red fluorescence (spearman rank correlation of 0.89) (figure 4B), but

237 poorly with the melting temperature of variants (Figure 4C). This observation suggests that at

238 the onset of protein synthesis the DnaK promoter is upregulated purely in response to increased

239 protein translation - independent of the stability of the expressed protein. Later in the time

240 course the rate of red fluorescence no longer corresponds to the rate of translation due to

241 increased influence of degradation, and the correlation between the relative rate of red and

242 relative rate of green fluorescence diminishes (Figure $4 \mathrm{H}$ ).

244 Variants with low stability show increased DnaK transcription

245 In contrast to what is observed at early rates, there is a moderate correlation between rate of

246 GFP and melting temperature following the first $60 \mathrm{~min}$ of protein synthesis. The correlation 
appears to increase at later timepoints (Figure 4D and 4G). While individual correlation values

248 fluctuate and are associated with statistical uncertainty, there is a clear indication that the DnaK

249 response is affected by the thermal stability of the variants (Figure 4G). Notably, after 120 min,

250 cells expressing the most stable variants (wt, Q33Y and V36I) experience a significant drop in

251 green fluorescence (Figure 4A+D). This could suggest that the stable variants do not engage

252 DnaK, likely due to lower concentration of unfolded/misfolded proteins. However,

concurrently with a drop of in green fluorescence cells expressing these three variants also

experience a decrease in OD. Consequently, the decrease in expression from the DnaK

promoter could be a trait associated with cell death. The green fluorescence in cells expressing

the remaining five variants continue to increase, although with reduced rates (Figure $4 A+D)$

and with higher rate of green fluorescence for the less stable variants. A correlation between

the subset of five with positive GFP rates, but only after 150 minutes (spearman rank correlation 0.78 for rates calculated in the range $180-210$ minutes and 0.64 for the $210-240$ interval). So, as could be expected, our assay indicates an increased requirement for chaperone assistance for the less stable variants.

263 The first 50 min post-induction there is a moderate correlation between melting temperature and accumulated green fluorescence. However, onwards from 60 min the correlation decreases steadily (4F). This most likely reflects the variation in protein abundance as the effect of

266 degradation of the N102LT variants becomes increasingly pronounced. To study how much

267 the expression and stress response vary between individual cells, we must consider differences in plasmid copy number in cells. Since both RFP and GFP are expressed from the same plasmid 
272 (Figure 5A, S5). After that point, the normalised DnaK response decreases in a manner highly

273 dependent on the expressed variant (Figure 5B). In addition, the accumulated and normalised

274 DnaK response correlates strongly with the melting temperature of the variant following the

275 initial peak at $30 \mathrm{~min}$ and throughout the entire $240 \mathrm{~min}$ (Figure 5D). This is exemplified by

276 the 120-minute time point, where we see a clear distinction between the chaperone response

277 triggered for the eight N102LT variants, with a strong negative correlation between the green

278 to red fluorescence ratio and the melting temperature of the expressed variants (Figure 5C).

279 Less stable variants induce a stronger DnaK response, and that pattern persists over time. This

280 observation is a strong indication that the less stable variants require continuous assistance

281 from PQC chaperones to maintain their native fold, whereas more stable variants do not.

282

283

Expression of the wt, Q33Y and V36I variants leads to occurrence of subpopulations with

\section{altered fluorescence phenotypes}

The above analysis shows that evaluation of protein abundance as well as DnaK response gives

a good insight into the effect of protein biophysical properties on whole populations of cells expressing the same variant. However, with flow cytometry we can go beyond the study of cells at the population level and investigate the cell-to-cell variation in protein expression and

DnaK response. To fully explore the phenotypic variation within a population, we cluster the cells based on red and green fluorescence using a Bayesian Gaussian mixture model. Although not all identified clusters may represent homogenous phenotypes, automated clustering allows

292 for unbiased assignments. Figure 6 shows the clusters identified for variant L57G and V36I at times 0,90 , and $240 \mathrm{~min}$ in a double logarithmic plot. If the cells form a straight line with a

294 slope of one in the double logarithmic plot the green to red fluorescence ratio is constant in all cells and is equivalent to the intersection with the y-axis. A slope below one indicates that the variation in red fluorescence is larger than the variation in green fluorescence, whereas a slope 
297 above one indicates that the variation in green fluorescence is bigger than the variation in red

298 fluorescence.

299 For all variants we identify one main population consisting of 3 to 4 different clusters (Figure

300 6, S6-7). The distribution of red and green fluorescence within the main population differs

301 between variants and with time. At the time of induction, both green, and especially red

302 fluorescence, show significant cell-to-cell variation for all variants. This is evident by the

303 dispersed distribution around a line with a slope below one and is observed regardless of

304 variant. However, for some variants there is an indication of a diagonal line pre-induction. This

305 is likely due to leaky expression. Once recombinant protein synthesis is induced, the

306 distribution of red and green fluorescence in the main population becomes increasingly

307 homogenous and approaches a near-constant ratio between green and red fluorescence for all

308 cells in a population (Figure 6, Figure 7, S6-8). Interestingly, when expressing variants with a

309 melting temperature below the wt, reaching the near-constant ratio is significantly delayed

310 (Figure 7, S8).

312 For the least stable variants (L57G, L57C, L57P and M40A) and Q33S, with a stability close

313 to wt, there appears to be only a single population (Figure 6+S6-7). For wt and the two most

314 stable variants (Q33Y and V36I), however, individual cells with less protein abundance (lower

315 red fluorescence intensity) emerge after $120 \mathrm{~min}$, and the fraction of these cells increase over

316 time. After 240 minutes, distinct populations with phenotypic variation in DnaK response can

317 be observed for cells expressing wt, Q33Y and V36I. Cells with low green fluorescence have

318 either low or high red fluorescence, forming two distinct populations. We note that the

319 emergence of subpopulations with altered fluorescence phenotypes are highly variant-specific

320 and is only observed for cells expressing the three most stable variants and concur with a

321 reduction in OD for cells expressing these variants. Neither formation of subpopulations nor 
reduction in OD is observed for cells expressing Q33S which has thermostability equivalent to the wt.

\section{Morphological changes are observed for E. coli expressing N102LT variants}

326 Stressed cells often show morphological changes. We monitored cell morphology by forward

327 (FSC) and side scatter (SSC) to identify potential variant-dependent changes. FSC provides

328 information about the size of the cell, and for most variants there is no change in FSC over the

329 four-hour time-course indicating that the size of the cells remains constant (Figure 8A, S9).

330 However, for three variants (wt, Q33Y and V36I) the FSC increases after 120 minutes,

331 indicating an increase in cell size. Furthermore, these three variants are distinctly different from

332 the other five variants throughout the timeseries, indicating that cells expressing wt, Q33Y and

333 V36I might be bigger even prior to induction. For eukaryotic cells SSC reports on the

334 complexity of the cell but it remains unclear how to interpret SSC from bacterial cells, although

335 it has been suggested that SSC is associated with cell wall density [36]. We observe a variant-

336 dependent decrease in SSC during the first two hours after induction Figure 8B, S10) with a

337 moderate negative correlation to the melting temperature of the expressed variant. Although

338 the implications of SSC are not understood it is evident from the patterns of FSC and SSC

339 variation that cells undergo morphological changes, and that the changes depend on the 340 expressed variant.

\section{Discussion}


346 We have demonstrated that DnaK expression is activated immediately once recombinant

347 protein expression starts. It then shifts from being dependent on protein translation rates to

348 being primarily governed by protein stability at later time points. The initial, stability-

349 independent activation is most likely due to the increase of unfolded protein as the nascent

350 peptide chain exits the ribosome. The observed correlation between onset of protein synthesis

351 and initial DnaK response can be caused by either co-translational binding of DnaK to the

352 emerging peptide chain or binding of DnaK to newly synthesised, unfolded, protein after it is

353 released from the ribosome (Figure 9). However, several $\lambda$-repressor constructs have been

354 shown to fold in submilisecond time scales [37-39] why presence of significant amounts of

355 unfolded $\lambda$-repressor are unlikely. Binding of DnaK to the unfolded protein releases DnaK-

356 bound $\sigma^{32}$ which acts as transcription factor for synthesis of DnaK as well as other PQC

357 proteins. The consequence is an increase in transcription of PQC proteins that are unrelated to

358 protein thermostability but tightly linked to translation rate. Besides aiding in nascent chain

359 folding, this linkage adjusts the amount of PQC network components to the overall protein

360 expression level and thus ensures that enough chaperones are present to deal with potential

361 protein misfolding. The rate of GFP synthesis as well as accumulated GFP/RFP signal

362 correlates with variant melting temperature after the initial 30 minutes of protein synthesis.

363 This correlation suggests that the DnaK response is impacted by the proportion of protein in

364 the unfolded/misfolded state at folding equilibrium (Figure 9). Hence, variants with lower

365 stability require increased DnaK activity compared to more stable variants. Increased

366 interaction with DnaK increases the degree of degradation by proteases, as DnaK, given its

367 role as a central hub in the PQC network, is involved in targeting misfolded proteins for

368 degradation [40]. As a consequence, in our study, most protein is accumulated in cells

369 expressing the four variants that are at least as stable as the wt (wt, Q33S, Q33Y and V36I),

370 and thus does not require continuous assistance from chaperones. 
372 Analysis of the accumulated DnaK response and N102LT variant accumulation in single cells

373 revealed that cell-cell variation, especially in protein accumulation, is bigger early after onset

374 of protein synthesis and decreases with time. There is a prolonged period with a wide

375 distribution in protein accumulation observed for variants less stable than wt. This lag can

376 potentially be explained by increased degradation: The variation in protein abundance can be

377 caused by a variance in both protein synthesis and degradation. The variance for the more stable

378 variants, however, is primarily due to variation in synthesis. Once folding and

379 synthesis/degradation equilibrium is reached, a single population of cells is seen, with similar

380 chaperone response relative to protein abundance. This suggests that as the cells reach some sort of homeostasis, the variation in DnaK response can be largely explained by variation in accumulated protein.

383

384 However, for the wt, Q33Y and V36I variants subpopulations with reduced DnaK response emerges after approximately 120 minutes. In association with the emergence of subpopulations in these three stable variants there is a decrease in $\mathrm{OD}_{600}$ and $\mathrm{DnaK}$ as well as an increase in cell size (a phenotype often associated with stressed cells [41]) is observed. The transformation into these alternative phenotypes is potentially related to the burden associated with a high protein load in the cell. Despite the Q33S reaching the highest overall abundance of protein, this variant does not share the described phenotype indicating a more complex relationship between protein stability and cellular response. 
395 Although we find a good overall correlation between the variant melting temperature and the

396 DnaK response after expression in E. coli, not all the variation can be explained by the altered

397 thermostability. Consequently, there might be other sequence-dependent effects that we have

398 not investigated further. The folding equilibrium might be shifted in vivo compared to the

399 conditions in vitro. The unfolded state for instance, could be stabilized by inadvertent

400 interactions with other macromolecules. Similarly, the folded state could be further stabilised

401 by non-specific interactions, e.g. with DNA. Altered affinity for DNA could also affect a

402 variant's toxicity and potentially explain the altered phenotypes observed exclusively for the

403 wt, Q33Y and V36I which cannot be explained by thermostability alone. The DnaK-dependent

404 signal is further potentially skewed by a variant's aggregation propensity as aggregation itself has an impact on PQC network activity [14].

406

\section{Differential PQC response could constrain evolution of protein sequences}

408 Several studies have investigated the coupling between biophysical properties and protein evolution. A strong negative correlation between rates of protein coding sequence evolution

410 and gene expression levels have been observed [11, 42]. This has been coupled to protein

411 stability by two different models. In the first, unstable proteins have a lower concentration of

412 active protein resulting in lower fitness. In the second, the misfolded proteins are toxic to the

413 cell with an associated fitness cost. Mathematically, these models become identical for proteins

414 with stabilities beyond $5 \mathrm{kcal} / \mathrm{mol}$ [43]. Geiler-Samerotte et al. [14] directly tested the

415 misfolding hypothesis by evaluating growth rates in yeast expressing misfolded proteins. They

416 found a significant fitness cost due to misfolded proteins and associated upregulation of HSP70

417 (DnaK). The increased evolutionary rate of proteins with stronger binding to DnaK suggests

418 that chaperones can buffer deleterious mutations [10]. Nonetheless, triggering the PQC

419 network comes with a cost and it is possible that this burden on the cell can impact selection 
420 of protein variants during evolution. The observation that small amino acid changes can have

421 a significant impact on the chaperone response thus suggests that the degree of $d n a K$-activation

422 by a protein may itself act as a significant selection factor. The exact relation between

423 magnitude of the PQC and fitness, however, is not clear. In our experiments, we did not observe

424 a correlation between DnaK response and cell growth rates, but such correlations are likely

425 obscured by variations in protein abundance. Moreover, the phenotypic differences can be very

426 small to still have an effect on evolutionary time-scales. It should be noted that we carry out

427 our experiments under conditions of strong protein overexpression. Hence, it is not clear if our

428 results are relevant to evolution of protein sequences under more standard conditions.

430 The reporter plasmid has potential use for screening protein stability

431 Stability is often a prerequisite for the application of proteins in research, medicine and

432 biotechnology. The development of accurate and high-throughput assays that can screen for 433 stability independent on protein function are thus desired. Our data demonstrate strong 434 correlations between in vitro protein stability and fluorescence values recorded from single cells expressing the N102LT variants. This highlights the utility of the fluorescent reporter plasmid in combination with flow cytometry analysis as an assay for screening for protein stability in vivo. We propose two potential approaches for using the reporter assay for screening: (i) using the GFP/RFP ratios of tagRFP fusion proteins as a high throughput screen

439 for variant libraries, e.g. for directed evolution. The strong correlation between GFP/RFP ratio

440 and thermostability provides optimal conditions for screening for variants with increased 441 stability since the correlation varies minimally with time. (ii) using the rate of GFP for high442 throughput stability screening of protein variants. This approach is potentially less robust than 443 the GFP/RFP ratio method but has the advantage of not requiring the proteins of interest to be 444 fused to tagRFP. This can for example be beneficial in cases where the studied protein is 
446 assembly.

\section{Material and methods}

\section{$448 \quad$ Strains and plasmids}

449 A modified pQlinkN plasmid [44] were created by inserting additional restriction sites for PstI, 450 SacII and NdeI between the Link1-region and the multiple cloning site (MCS) of pQlinkN. The 451 genes encoding sfGFP and the dnaK-promoter $\left(\mathrm{P}_{\text {DnaK }}\right)$ was amplified by PCR from E.coli 452 BL21*(De3) genomic DNA, respectively. Both $s f g f p$ and $P_{\text {dnak }}$ was inserted into the modified 453 pQlinkN plasmid using restriction enzyme cloning.

The gene for N102LT N-terminally fused with tagRFP (gi: 336287738) was synthesised (GenScript) and inserted to unmodified pQlinkN plasmid using restriction enzyme cloning. Point mutations in the N102LT gene were made by site-directed mutagenesis with Phusion HotStart II DNA polymerase to create seven N102LT variants: Q33S, Q33Y, V36I, M40A, L57C, L57G and L57P. The full reporter plasmid was created by ligation independent cloning with pQLinkN harbouring N102LT-tagRFP variants as recipient vector and modified pQlinkN harbouring $P_{D n a K}$ and $s f-g f p$ as insert vector [44].

462

\section{Protein expression}

464 E. coli BL21(DE3) pLysS was transformed with reporter plasmid harbouring the N102LT 465 variants for expression. Cells were grown at $37{ }^{\circ} \mathrm{C}$ in $\mathrm{LB}$ medium with shaking (150 rpm) and 466 were induced with $1 \mathrm{mM}$ isopropyl- $\beta$-d-thiogalactopyranoside (IPTG) when cells reached an

$467 \mathrm{OD}_{600}$ between 0.4 and 0.6 and continued to grow for four hours. Variants were expressed on 
separate days, but both replicates of the one variant was expressed individually on the same

469 day.

\section{$471 \quad$ Flow cytometry}

472 Culture samples were taken just before induction with IPTG (time point $0 \mathrm{~min}$ ), after $2 \mathrm{~min}$, every five min the first $30 \mathrm{~min}$, every $10 \mathrm{~min}$ from $30 \mathrm{~min}$ to $120 \mathrm{~min}$ and every half hour until 240 min after induction. Immediately after taken the sample it is diluted diluted 1:1000 in 10 $\mathrm{mM} \mathrm{Na}_{2} \mathrm{HPO}_{4}, 1.8 \mathrm{mM} \mathrm{KH}_{2} \mathrm{PO}_{4}, 137 \mathrm{mM} \mathrm{NaCl}, 2.7 \mathrm{mM} \mathrm{KCl} \mathrm{pH}=7.4$ and analysed on s3e cell sorter (Biorad). Replicates were measured immediately after each other giving a time lag between replicates of 10-30 sec. 3000 events were collected with an event rate of $\sim 500$ events/s. A laser with $488 \mathrm{~nm}$ was used for detection. Cells expressing N102LT variants were gated on forward and side scatter to exclude doublets. Fluorescence was detected using a 525/30 filter

480 (green fluorescence) and a 615/25 filter (red fluorescence). Flow cytometry data was analysed using the flowCore [45], flowViz [46] and flowWorkspace [47] packages within the Bioconductor frame work.

\section{Clustering}

485 Flow cytometry data was clustered using Bayesian Gaussian mixture model, implemented in scikit-learn [48]. Up to 7 different components were allowed and priors for weights were

487 described by a Dirichlet distribution with a weight concentration prior of 1e-6. A diagonal 488 covariance matrix was used for each component.

\section{Data analysis}


491 Mean red and green fluorescence as well as mean forward and side scatter intensities were

492 calculated from the absolute intensity for each event for a given variant at a given time. Mean

493 green/red fluorescence intensity was calculated by taking the mean over the green/red

494 fluorescence intensity for each variant for each timepoint. Rates were estimated by fitting a

495 linear trendline to the mean red, green or green/red fluorescence intensity within the indicated

496 time interval. Relative rates were estimated by normalizing the rate of fluorescence with the

497 mean fluorescence within the same time interval. Mean red, green and green/red fluorescence

498 as well as relative rates were calculated individually for each replicate. Spearman correlations

499 between red, green or green/red and variant melting temperature were calculated based on both

500 replicates of each variant. A linear trend line was fitted to the $\log 10$ transformed red and green

501 fluorescence for each event for a given variant at any given time point. A trend line was fitted

502 individually for each replicate.

503

504 Acknowledgements

505 We would like to thank Dr. Fabian Cornejo for helpful comments to the manuscript. We

506 would also like to thank Robert T. Sauer for providing the original N102LT construct.

508 Author contributions

509 Sebastian Rämisch: Methodology, Writing - Review and editing

510 Signe Christensen: Conceptualization, Methodology, Formal analysis, Investigation, Writing

511 - original draft, Visualization

512 Ingemar André: Conceptualization, Methodology, Software, Resources, Supervision, Writing

513 original draft 
516 The authors declare that they have no conflict of interest.

517

\section{Funding}

519 This work was supported by the European Research Council (ERC) under the European Union's

520 Horizon 2020 research and innovation programme [771820]

521

\section{References}

523 1. Rudiger, S., A. Buchberger, and B. Bukau, Interaction of Hsp70 chaperones with 524 substrates. Nat Struct Biol, 1997. 4(5): p. 342-9.

525 2. Guisbert, E., et al., A chaperone network controls the heat shock response in E. coli. Genes Dev, 2004. 18(22): p. 2812-21.

3. Blaszczak, A., C. Georgopoulos, and K. Liberek, On the mechanism of FtsHdependent degradation of the sigma 32 transcriptional regulator of Escherichia coli and the role of the Dnak chaperone machine. Mol Microbiol, 1999. 31(1): p. 157-66.

530 4. Plata, G., M.E. Gottesman, and D. Vitkup, The rate of the molecular clock and the cost of gratuitous protein synthesis. Genome Biol, 2010. 11(9): p. R98.

5. Parsell, D.A. and R.T. Sauer, Induction of a heat shock-like response by unfolded protein in Escherichia coli: dependence on protein level not protein degradation. Genes Dev, 1989.3(8): p. 1226-32.

535 6. Sekhar, A., H.N. Lam, and S. Cavagnero, Protein folding rates and thermodynamic stability are key determinants for interaction with the Hsp70 chaperone system.

538 7. Calloni, G., et al., DnaK functions as a central hub in the E. coli chaperone network. Cell Rep, 2012. 1(3): p. 251-64. 
540 8. Thomas, J.G. and F. Baneyx, Protein misfolding and inclusion body formation in recombinant Escherichia coli cells overexpressing Heat-shock proteins. J Biol Chem, 1996. 271(19): p. 11141-7.

9. Hoffmann, F. and U. Rinas, Kinetics of heat-shock response and inclusion body formation during temperature-induced production of basic fibroblast growth factor in high-cell-density cultures of recombinant Escherichia coli. Biotechnol Prog, 2000. 16(6): p. 1000-7.

10. Kadibalban, A.S., et al., DnaK-Dependent Accelerated Evolutionary Rate in Prokaryotes. Genome Biol Evol, 2016. 8(5): p. 1590-9.

11. Drummond, D.A., A. Raval, and C.O. Wilke, A single determinant dominates the rate of yeast protein evolution. Mol Biol Evol, 2006. 23(2): p. 327-37.

12. Drummond, D.A. and C.O. Wilke, Mistranslation-induced protein misfolding as a dominant constraint on coding-sequence evolution. Cell, 2008. 134(2): p. 341-52.

13. Dong, H., L. Nilsson, and C.G. Kurland, Gratuitous overexpression of genes in Escherichia coli leads to growth inhibition and ribosome destruction. J Bacteriol, 1995. 177(6): p. 1497-504.

14. Geiler-Samerotte, K.A., et al., Misfolded proteins impose a dosage-dependent fitness cost and trigger a cytosolic unfolded protein response in yeast. Proc Natl Acad Sci U S A, 2011. 108(2): p. 680-5.

15. Rutherford, S.L. and S. Lindquist, Hsp90 as a capacitor for morphological evolution. Nature, 1998. 396(6709): p. 336-42. Biol, 2008. 4(2): p. e1000002.

563 17. Bogumil, D., et al., Chaperones divide yeast proteins into classes of expression level and evolutionary rate. Genome Biol Evol, 2012. 4(5): p. 618-25. 
565 18. Wyganowski, K.T., M. Kaltenbach, and N. Tokuriki, GroEL/ES buffering and compensatory mutations promote protein evolution by stabilizing folding intermediates. J Mol Biol, 2013. 425(18): p. 3403-14.

19. Agozzino, L. and K.A. Dill, Protein evolution speed depends on its stability and abundance and on chaperone concentrations. Proc Natl Acad Sci U S A, 2018. 115(37): p. 9092-9097.

20. Williams, T.A. and M.A. Fares, The effect of chaperonin buffering on protein evolution. Genome Biol Evol, 2010. 2: p. 609-19.

21. Lachowiec, J., et al., Hsp90 promotes kinase evolution. Mol Biol Evol, 2015. 32(1): p. 91-9.

22. Moreau, M.J., I. Morin, and P.M. Schaeffer, Quantitative determination of protein stability and ligand binding using a green fluorescent protein reporter system. Mol Biosyst, 2010. 6(7): p. 1285-92.

23. Waldo, G.S., et al., Rapid protein-folding assay using green fluorescent protein. Nat Biotechnol, 1999. 17(7): p. 691-5.

24. Matreyek, K.A., et al., Multiplex assessment of protein variant abundance by massively parallel sequencing. Nat Genet, 2018. 50(6): p. 874-882.

25. Arsene, F., T. Tomoyasu, and B. Bukau, The heat shock response of Escherichia coli. Int J Food Microbiol, 2000. 55(1-3): p. 3-9.

584 26. Cluss, R.G., et al., Coordinate synthesis and turnover of heat shock proteins in Borrelia burgdorferi: degradation of DnaK during recovery from heat shock. Infect Immun, 1996. 64(5): p. 1736-43.

587 27. Mitchell, H.K., N.S. Petersen, and C.H. Buzin, Self-degradation of heat shock proteins. Proc Natl Acad Sci U S A, 1985. 82(15): p. 4969-73. 
589

590

591

592

593

594

595

596

598

599

600

601

602

603

604

605

606

607

608

609

610

611

612 38. Yang, W.Y. and M. Gruebele, Folding lambda-repressor at its speed limit. Biophys J, 613

28. Beamer, L.J. and C.O. Pabo, Refined 1.8 A crystal structure of the lambda repressoroperator complex. J Mol Biol, 1992. 227(1): p. 177-96.

29. Parsell, D.A. and R.T. Sauer, The structural stability of a protein is an important determinant of its proteolytic susceptibility in Escherichia coli. J Biol Chem, 1989. 264(13): p. 7590-5.

30. Lim, W.A. and R.T. Sauer, The role of internal packing interactions in determining the structure and stability of a protein. J Mol Biol, 1991. 219(2): p. 359-76.

31. Lim, W.A. and R.T. Sauer, Alternative packing arrangements in the hydrophobic core of lambda repressor. Nature, 1989. 339(6219): p. 31-6.

32. Lim, W.A., et al., The crystal structure of a mutant protein with altered but improved hydrophobic core packing. Proc Natl Acad Sci U S A, 1994. 91(1): p. 423-7.

33. Hecht, M.H., J.M. Sturtevant, and R.T. Sauer, Effect of single amino acid replacements on the thermal stability of the NH2-terminal domain of phage lambda repressor. Proc Natl Acad Sci U S A, 1984. 81(18): p. 5685-9.

34. Gutierres, M.B.B., C.B.C. Bonorino, and M.M. Rigo, ChaperISM: improved chaperone binding prediction using position-independent scoring matrices. Bioinformatics, 2020. 36(3): p. 735-741.

35. Balleza, E., J.M. Kim, and P. Cluzel, Systematic characterization of maturation time of fluorescent proteins in living cells. Nat Methods, 2018. 15(1): p. 47-51.

36. Diaz, M., et al., Application of flow cytometry to industrial microbial bioprocesses. Biochemical Engineering Journal, 2010. 48(3): p. 385-407.

37. Huang, G.S. and T.G. Oas, Submillisecond folding of monomeric lambda repressor. Proc Natl Acad Sci U S A, 1995.92(15): p. 6878-82. 2004. 87(1): p. 596-608. 
39. Burton, R.E., et al., The energy landscape of a fast-folding protein mapped by Ala--

40. Sherman, M. and A.L. Goldberg, Involvement of the chaperonin dnaK in the rapid degradation of a mutant protein in Escherichia coli. EMBO J, 1992. 11(1): p. 71-7.

41. Jeong, K.J. and S.Y. Lee, Enhanced production of recombinant proteins in p. $1295-8$.

42. Pal, C., B. Papp, and L.D. Hurst, Highly expressed genes in yeast evolve slowly.

43. Norn, C., I. Andre, and D.L. Theobald, A thermodynamic model of protein structure evolution explains empirical amino acid substitution matrices. Protein Sci, 2021.

44. Scheich, C., et al., Vectors for co-expression of an unrestricted number of proteins.

45. Ellis, B., Haaland, P., Hahne, F., Le Meur, N., Gopalakrishnan, N., Spidlen, J., version 1.38.2. 2016 .

46. Ellis, B., Gentleman., R., Hahne, F., Le Meur, N., Sarkar, D.,Jiang, M.,

47. Finak, G., Jiang, M., flowWorkspace: Infrastructure for representing and interacting with gated and ungated cytometry data sets. $R$ package version 4.4.0. 2021.

\section{Figure legends}


639 Figure 1 A fluorescent-based reporter system for protein abundance and DnaK response A) Overview of

640 reporter system: 1. Addition of IPTG allows for transcription of the GOI(Gene of Interest)-tagRFP by RNA

641 polymerase in complex with the transcription factor $\sigma^{70}$. 2. Following transcription of GOI-tagRFP the protein of

642 interest, POI-tagRFP is synthesised. 3. DnaK releases the transcription factor $\sigma^{32}$ and binds to unfolded/misfolded

643 POI-tagRFP. 4. $\sigma^{32}$ binds to RNA polymerase. 5. Binding of $\sigma^{32}$ to RNA polymerase allows for transcription of 644 endogenous PQC proteins, such as DnaK. The RNA polymerase - $\sigma^{32}$ complex will also bind to the DnaK 645 promoter on the reporter plasmid and transcribe the $s f G F P$ gene. 6. Following transcription of the $s f G F P$ gene, 646 the protein sfGFP will be synthesised. B) The N-terminal domain of the $\lambda$ repressor (blue) bound to DNA (purple).

647 Mutated residues shown as sticks (orange). PDB:11mb [28].

648 Figure 2 Growth of cells expressing N102LT variants A) $\mathrm{OD}_{600}$ as a function of time after induction of protein 649 synthesis for cells expressing the variants: L57G (dark blue), L57C (blue), L57P (cyan), M40A (green), Q33S 650 (yellow), V36I (orange), Q33Y (red) and wt (grey). One of two replicates are shown for each variant. B) Growth 651 rates prior to induction calculated for two replicates of each of the eight different N102LT variants with different 652 thermal melting temperatures. Colour scheme as in 2A. Spearman rank correlation $=0.14$. C) Growth rate the 653 first 120 min after induction calculated for two replicates of each of the eight N102LT-variants with different 654 melting temperature. Colour scheme as in 2A. Spearman rank correlation $=0.11$.

655 Figure 3 Abundance of tagRFP fusion proteins monitored by red fluorescence A) Mean red fluorescence 656 intensity of cells expressing variants: L57G (dark blue), L57C (blue), L57P (cyan), M40A (green), Q33S (yellow), 657 V36I (orange), Q33Y (red) and wt (grey). One of two replicates are shown for each variant. B) Spearman 658 correlation between mean red fluorescence and variant melting temperature at each time point. Calculations are 659 based on two replicates of each variant. Colour scheme as in 3A. C) Mean red fluorescence intensity at the 120 660 min time point for the eight N102LT variants with different melting temperature. Spearman correlation $=0.83$. 661 Colour scheme as in 3A. D) Early $(30-60 \mathrm{~min}$, black) and late $(60-90 \mathrm{~min}$, white) relative rates of red 662 fluorescence calculated for the eight N102LT variants with different melting temperature. Spearman correlation 663 between early rate and variant melting temperature is 0.05 . Spearman correlation between late rates and variant 664 melting temperature is 0.70 .

665 Figure 4 Transcription from the DnaK promoter monitored by green fluorescence A) Mean green 666 fluorescence intensity of cells expressing variants: L57G (dark blue), L57C (blue), L57P (cyan), M40A (green), 667 Q33S (yellow), V36I (orange), Q33Y (red) and wt (grey). One of two replicates are shown for each variant. B) 
The initial (0-30 min) relative rate of green fluorescence as a function of the initial rate of red fluorescence.

669 Spearman correlation $=0.89$. Both replicates shown. Colour scheme as in $4 \mathrm{~A}$ C) The initial $(0-30 \mathrm{~min})$ relative 670 rate of green fluorescence calculated for the eight N102LT variants with different melting temperature. Spearman 671 correlation between early rate and variant melting temperature is 0.40 . Colour scheme as in 4A. D) The relative 672 rate (150 $\mathrm{min}$ to $180 \mathrm{~min}$ ) of green fluorescence calculated for the eight N102LT variants with different melting 673 temperature. Spearman correlation between rate and variant melting temperature is 0.79 . Colour scheme as in

674 4A. E) Mean green fluorescence intensity at the $120 \mathrm{~min}$ time point for the eight N102LT variants with different 675 melting temperature. Spearman correlation between green fluorescence intensity and variant melting temperature 676 is 0.23 . Colour scheme as in 4A. F) Spearman correlation between accumulated mean green fluorescence and 677 variant melting temperature at each time point. Correlations are calculated based on both replicates of each variant.

678 G) Spearman correlation between rate of green fluorescence in the indicated time interval and variant melting 679 temperature. Correlations are calculated based on both replicates of each variant. H) Spearman correlation 680 between rate of green fluorescence and rate of rate fluorescence in the indicated time interval. Correlations are 681 calculated based on both replicates of each variant.

682 Figure 5 Accumulated transcription from the DnaK promoter normalised to protein abundance A) Mean 683 green fluorescence intensity divided by red fluorescence intensity of cells expressing variants: L57G (dark blue), 684 L57C (blue), L57P (cyan), M40A (green), Q33S (yellow), V36I (orange), Q33Y (red) and wt (grey). One of two replicates are shown for each variant. B) Relative rate of green/red fluorescence (30-60 $\mathrm{min})$ as a function of melting temperature. Spearman correlation $=0.55$ ). Colour scheme as in 5A C) Mean green fluorescence intensity divided by red fluorescence intensity at the 120 min time point for the eight N102LT variants with different melting temperature. Spearman correlation between green/red fluorescence intensity and variant melting temperature is 0.75 . Colour scheme as in 5A D) Spearman correlation between green/red fluorescence intensity and variant melting temperature at each time point. Calculations are based on both replicates of each variant.

691 Figure 6 Red and green fluorescence of single cells expressing L57G and V36I. The red and green fluorescence 692 for each individual cell in a population expressing L57G (top) and V36I (bottom) at the time of induction (left) and $90 \mathrm{~min}$ (middle) and $240 \mathrm{~min}$ (right) after induction with IPTG. Colouring in scatter plot is based on a

694 Bayesian Gaussian mixture model for cluster assignment. The x-axis histogram (red) shows the distribution of red fluorescence, and the y-axis histogram (green) shows the distribution of green fluorescence within the population. 
697 Figure 7 Slope of fluorescent population over time. For each time point the slope of log transformed red

698 fluorescence vs log transformed green fluorescence is calculated for L57G (dark blue) M40A (green), Q33Y (red)

699 and wt (grey). One of two replicates are shown for each variant.

700

701 Figure 8 Cell morphology of whole populations expressing N102LT variants. Forward (A) and side (B)

702 scatter as a function of time for variants: L57G (dark blue), L57C (blue), L57P (cyan), M40A (green), Q33S

703 (yellow), V36I (orange), Q33Y (red) and wt (grey). One of two replicates are shown for each variant.

704 Figure 9 A model for DnaK interaction with client proteins. Two different factors impact the interaction of

705 DnaK with client proteins: synthesis rate and protein stability. DnaK can act co-translationally with the nascent

706 peptide chain as it exits the ribosome exit tunnel, in which case DnaK response is expected to be proportional to

707 the translational rate. In addition, DnaK can bind to unfolded newly synthesised protein that has been released

708 from the ribosome. For slow folding protein the interaction with DnaK will then also depend on folding rate.

709 Fast folding protein will interact only minimally with DnaK. Fully synthesised proteins are in an equilibrium

710 between folded and misfolded determined by the stability of the protein. Thus, DnaK binding to misfolded

711 protein at folding equilibrium is dependent on protein stability 
A

Transcription of heat shock proteins

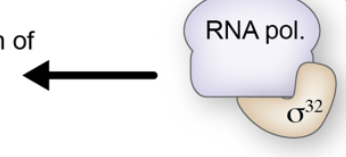

(5)
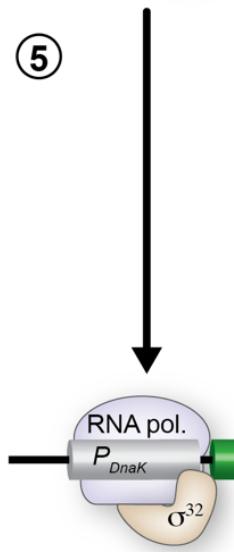

B

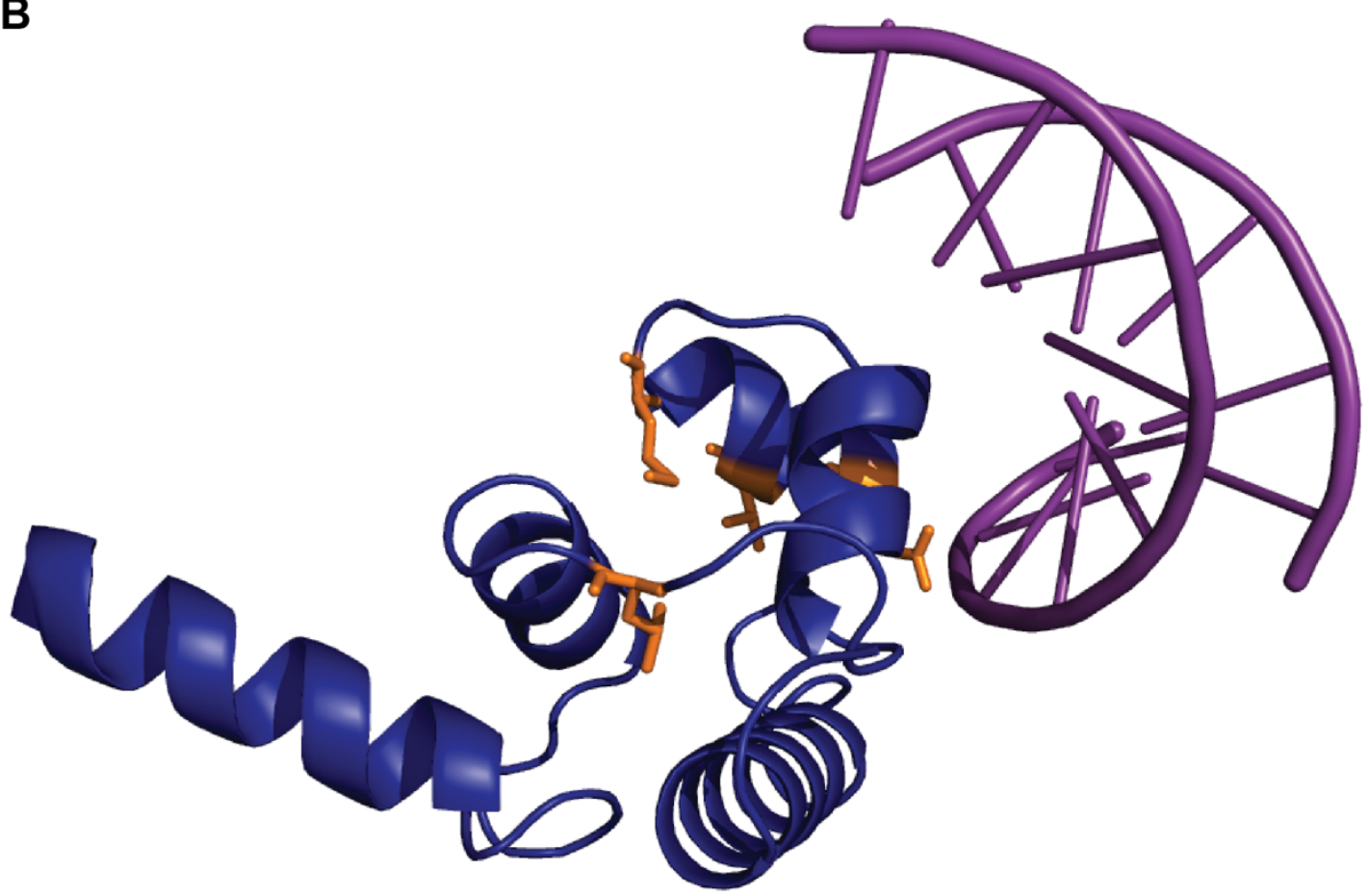

Figure 1 A fluorescent-based reporter system for protein abundance and DnaK response 


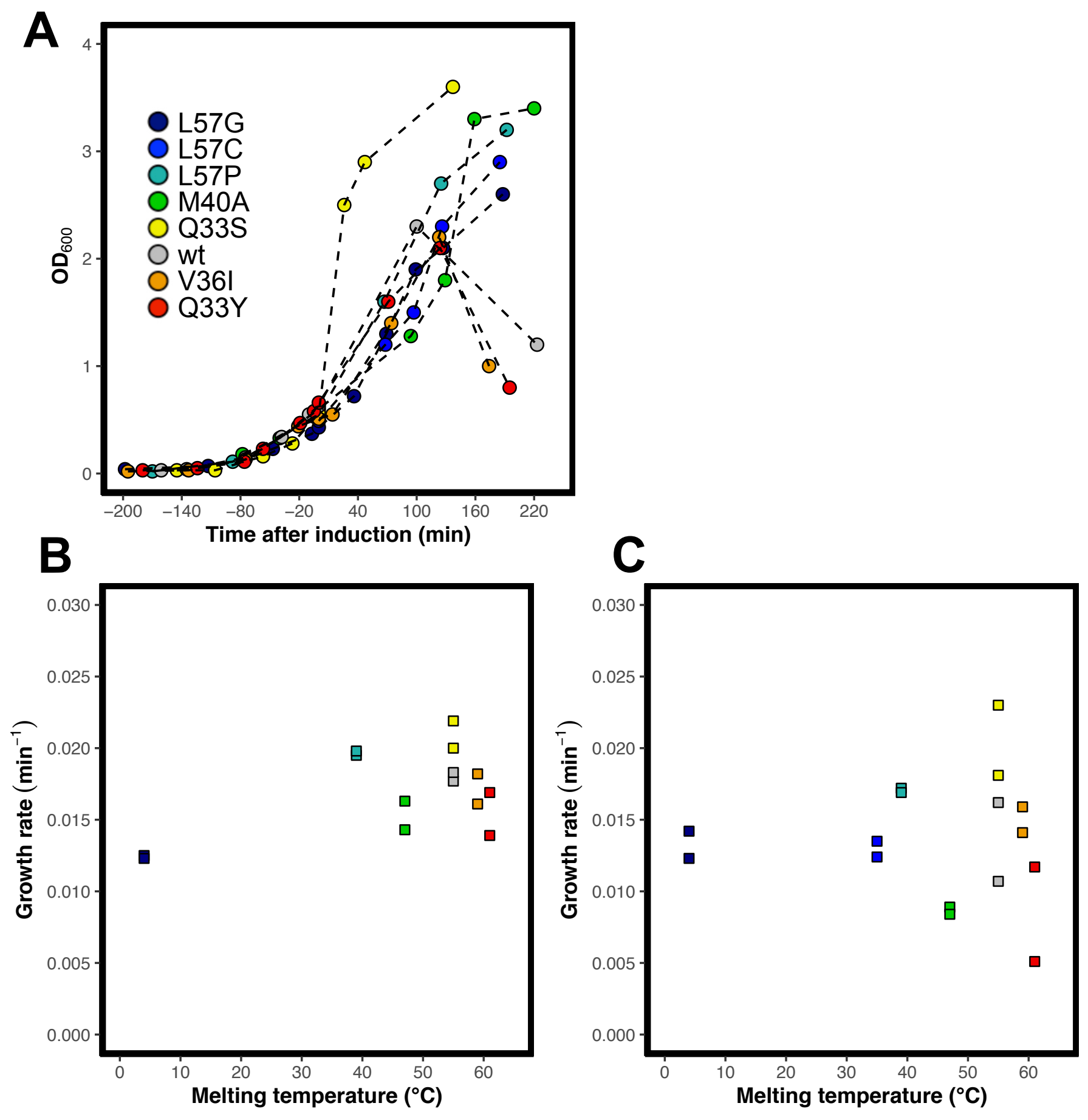

Figure 2 Growth of cells expressing N102LT variants 


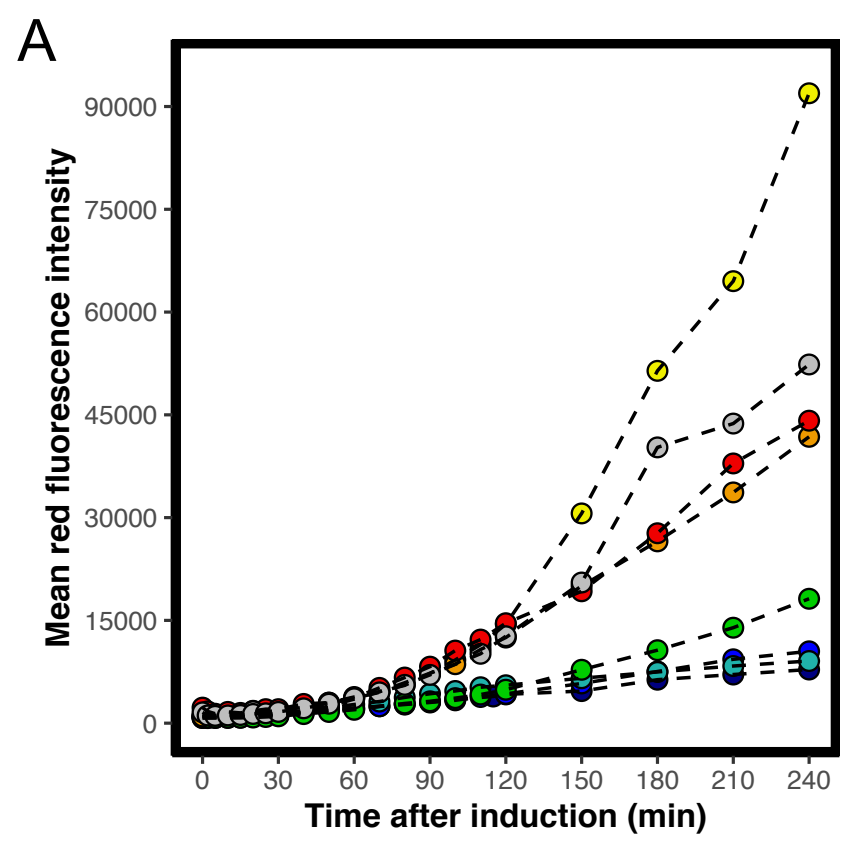

B

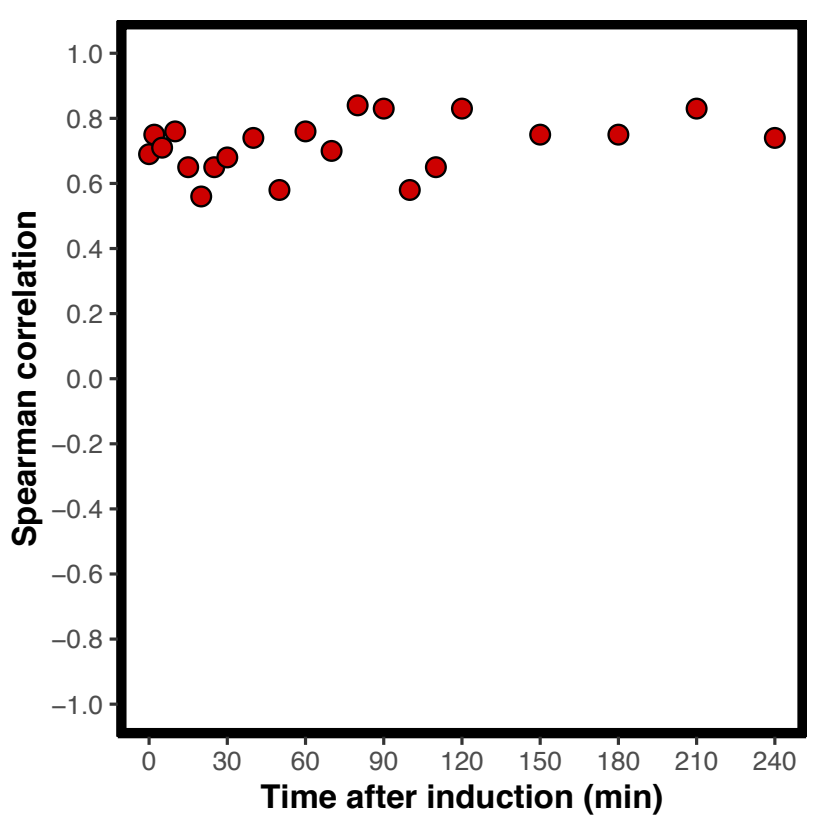

C

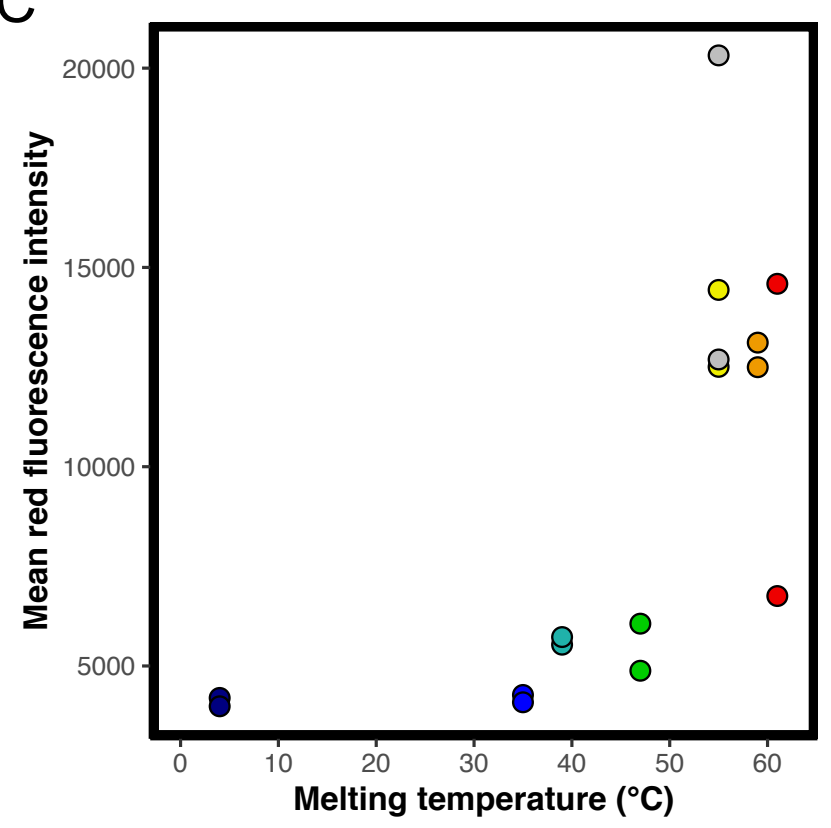

$\mathrm{D}$

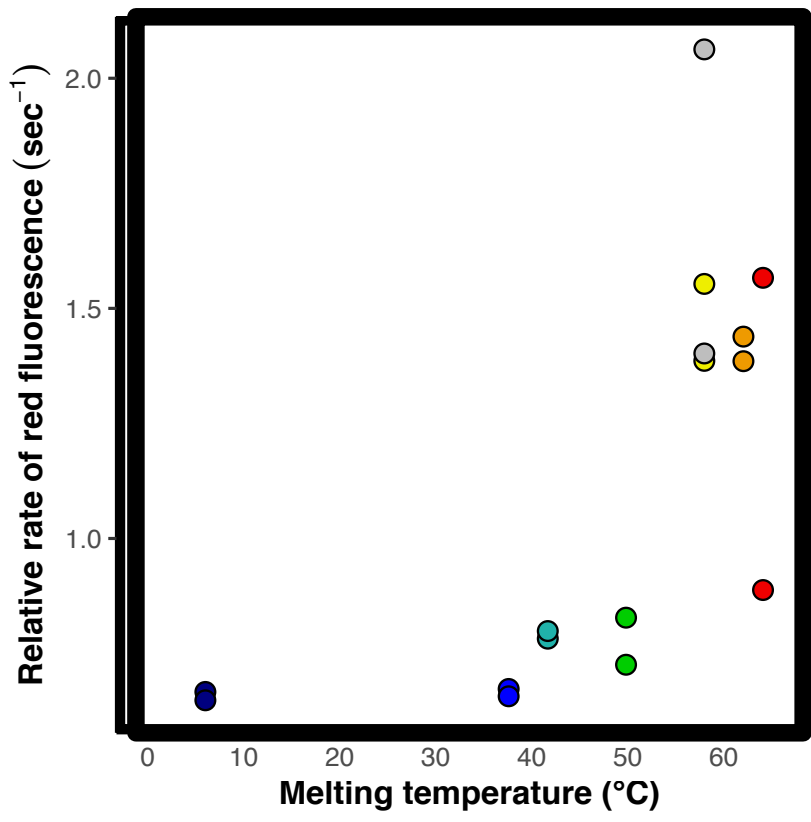

Figure 3 Abundance of tagRFP fusion proteins monitored by red fluorescence 
A

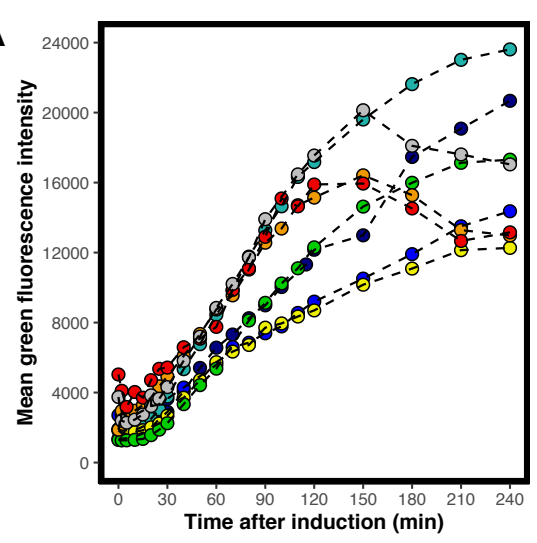

C

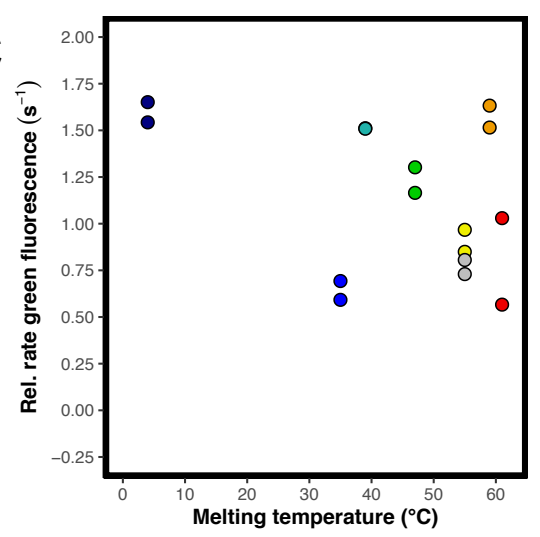

$\mathrm{E}$
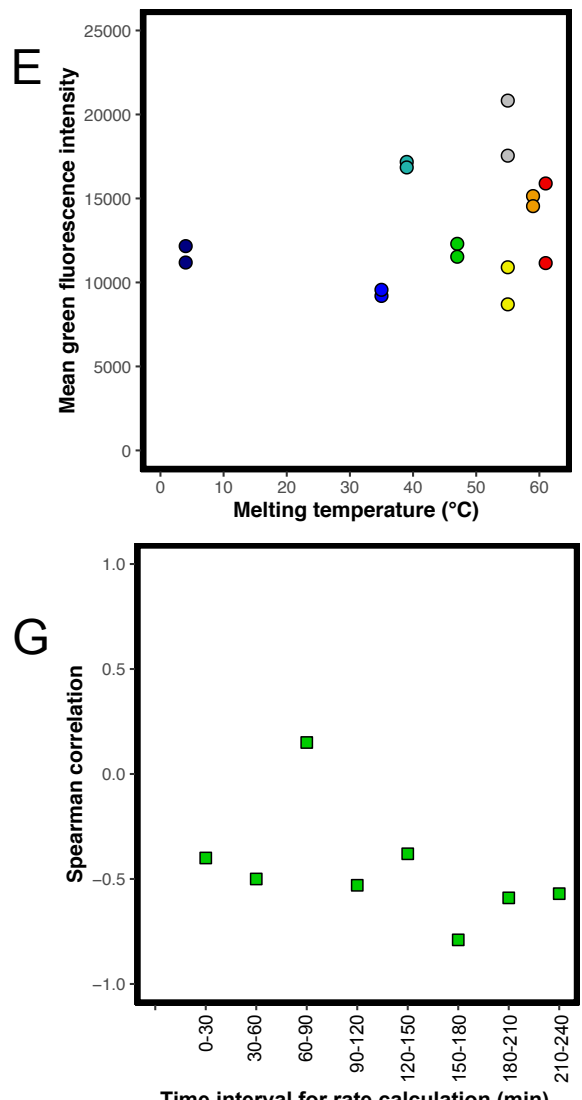

B
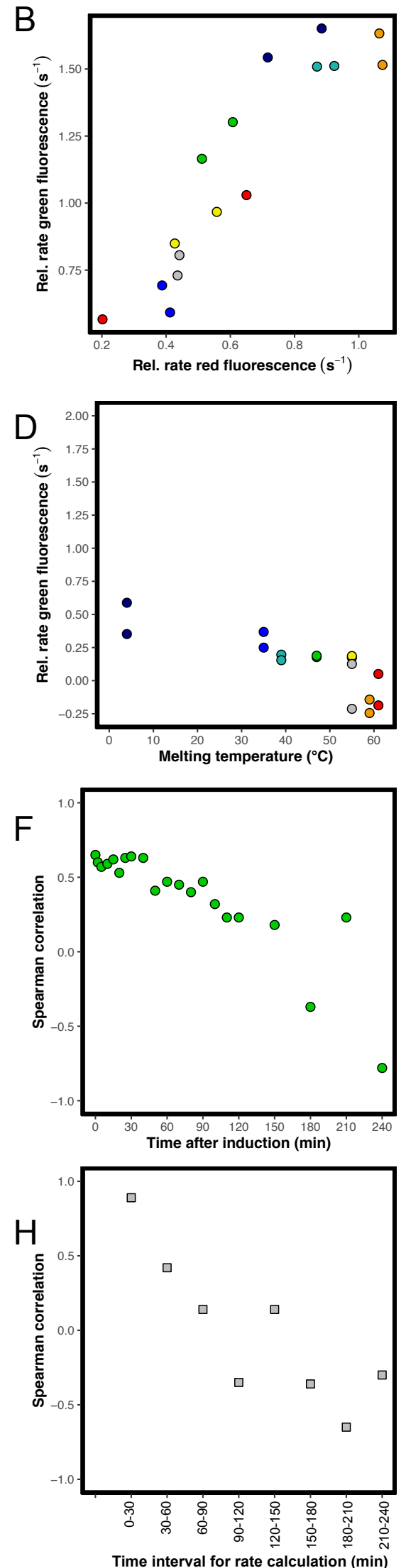

Figure 4 Transcription from the DnaK promoter monitored by green fluorescence 
A

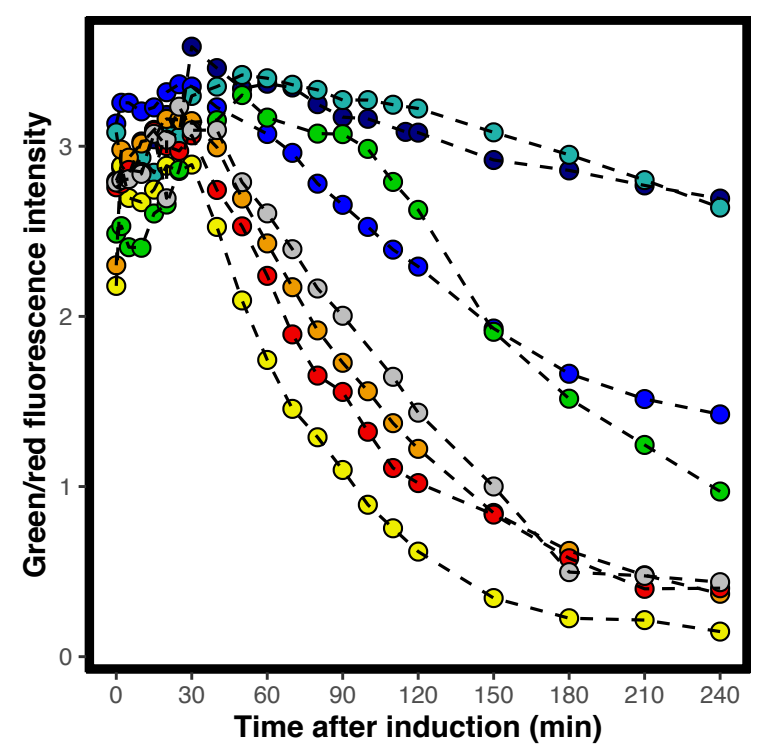

C

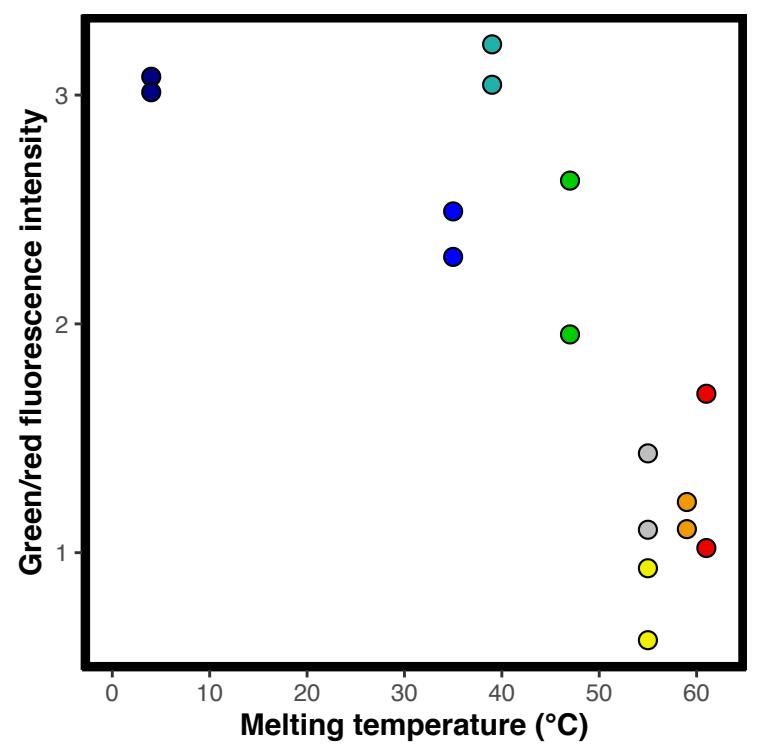

B

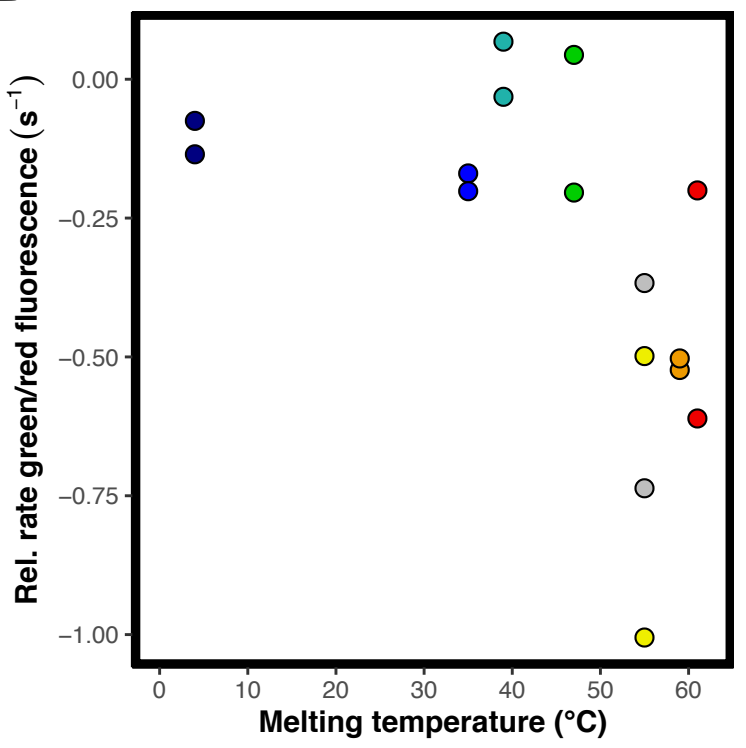

D

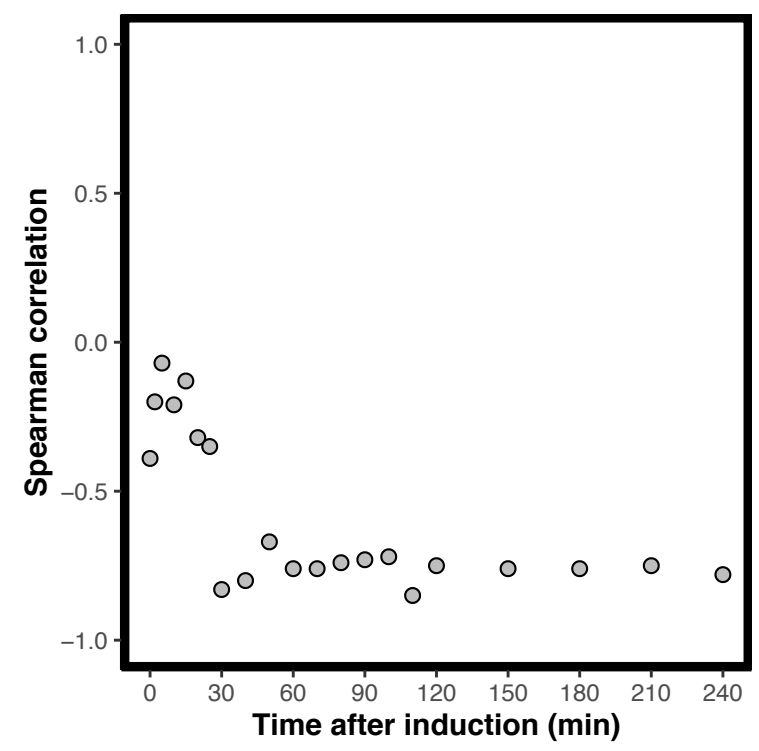

Figure 5 Accumulated transcription from the DnaK promoter normalised to protein abundance 

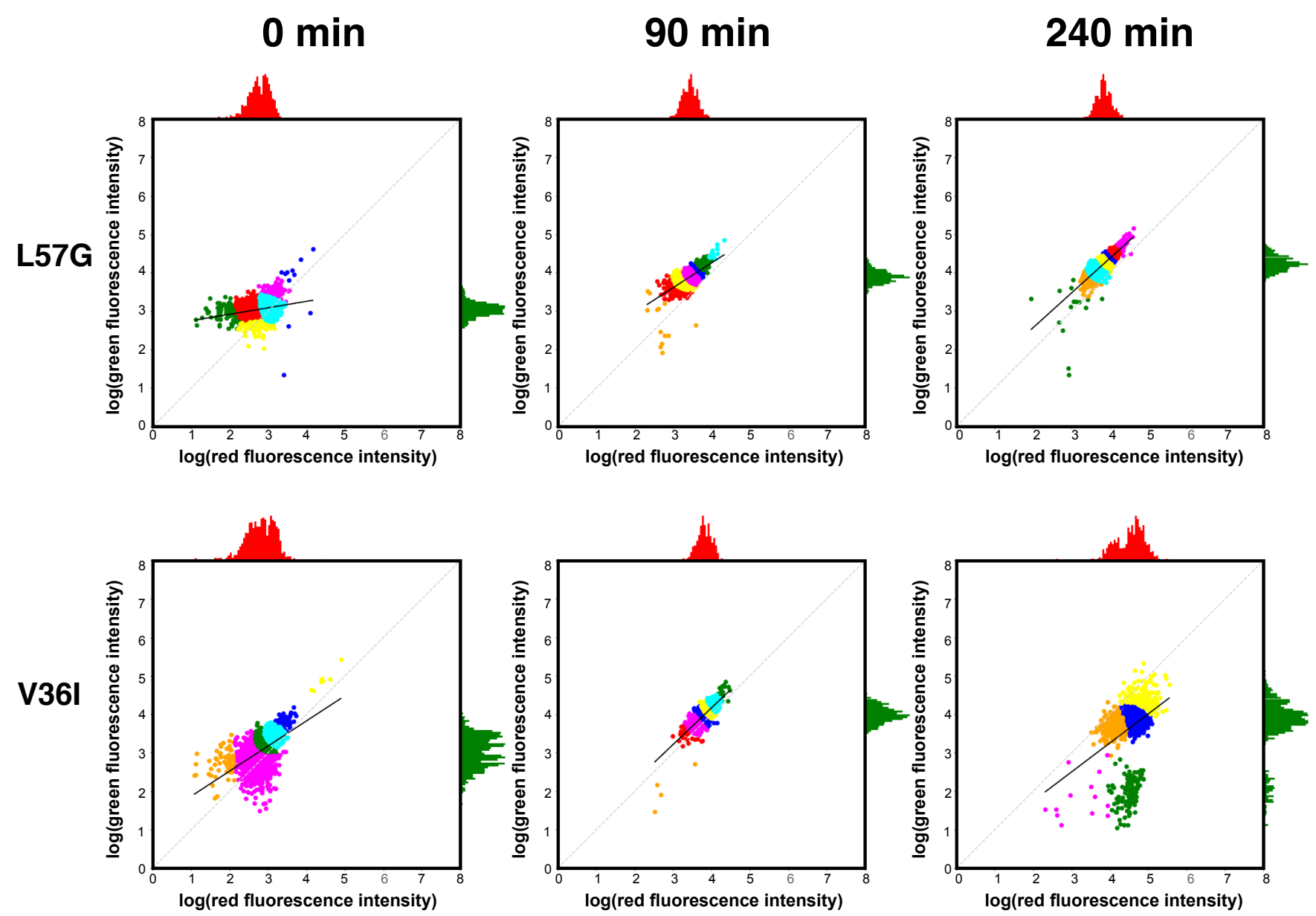

Figure 6 Red and green fluorescence of single cells expressing L57G and V36I. 


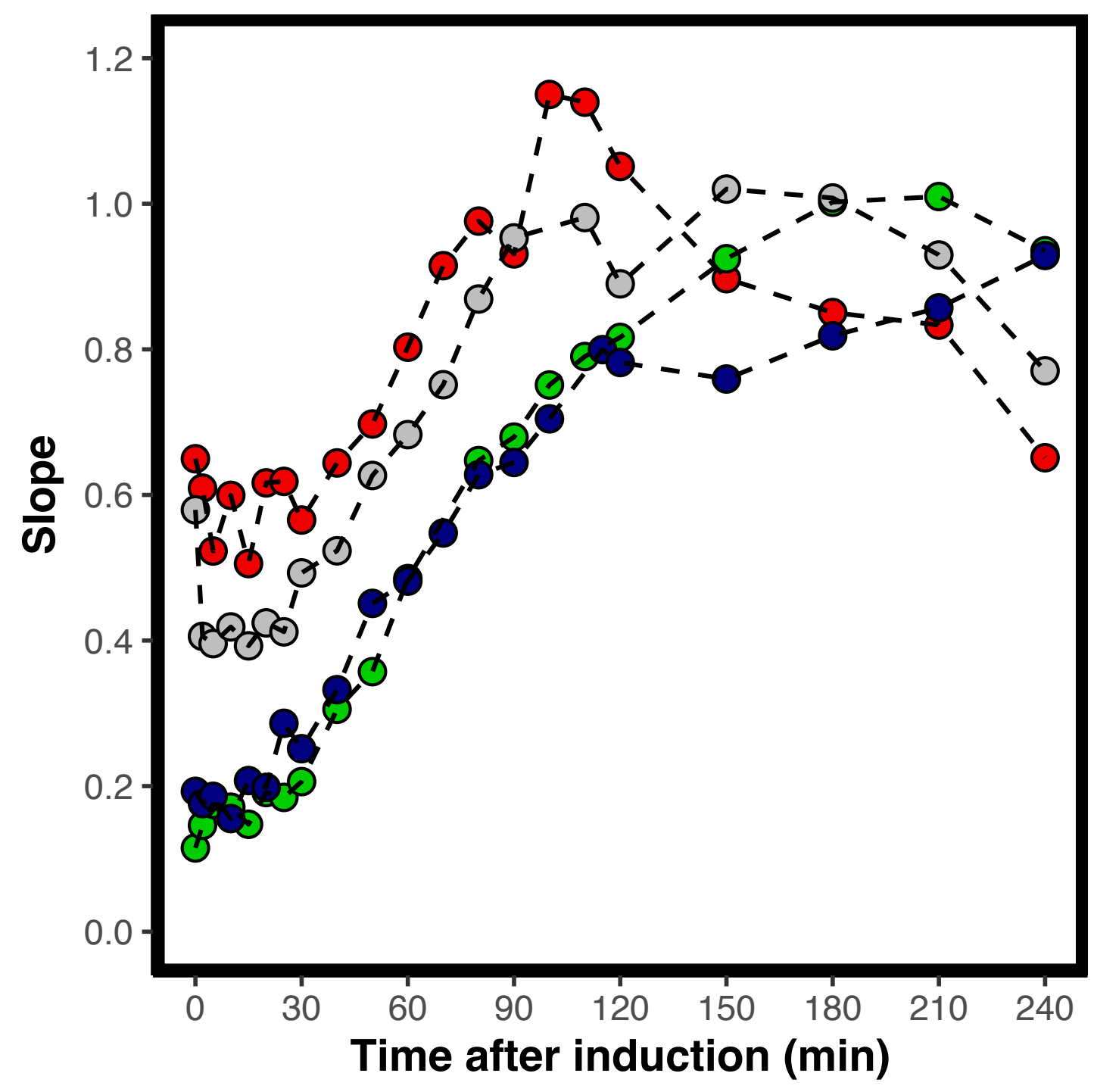

Figure 7 Slope of fluorescent population over time. 

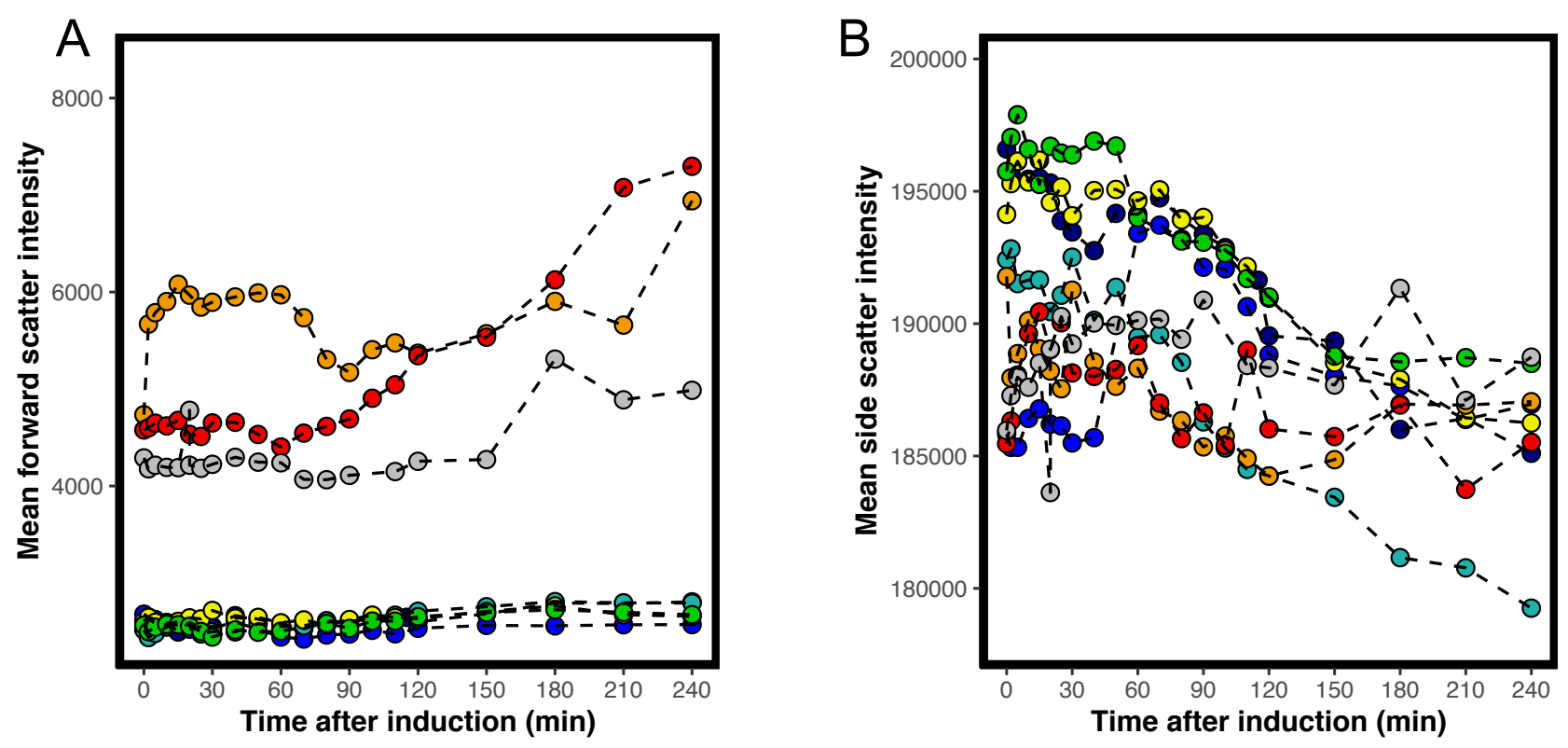

Figure 8 Cell morphology of whole populations expressing N102LT variants. 
bioRxiv preprint doi: https://doi.org/10.1101/2021.09.29.462496; this version posted October 1, 2021. The copyright holder for this preprint (which was not certified by peer review) is the author/funder, who has granted bioRxiv a license to display the preprint in perpetuity. It is made available under aCC-BY-ND 4.0 International license.

Synthesis-rate dependent DnaK binding

Co-translational binding of DnaK

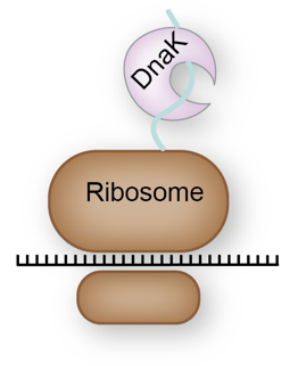

Interaction of DnaK with newly synthesised protein

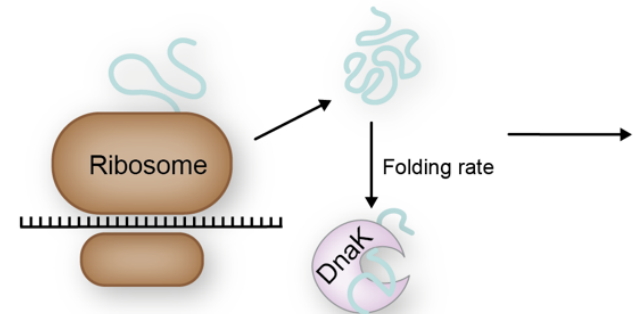

Stability-dependent DnaK-binding and degradation at folding equilibrium
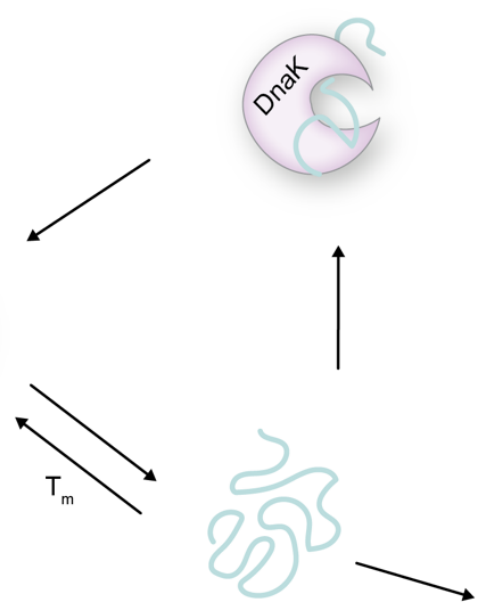

\section{Figure 9 A model for DnaK interaction with client proteins.}




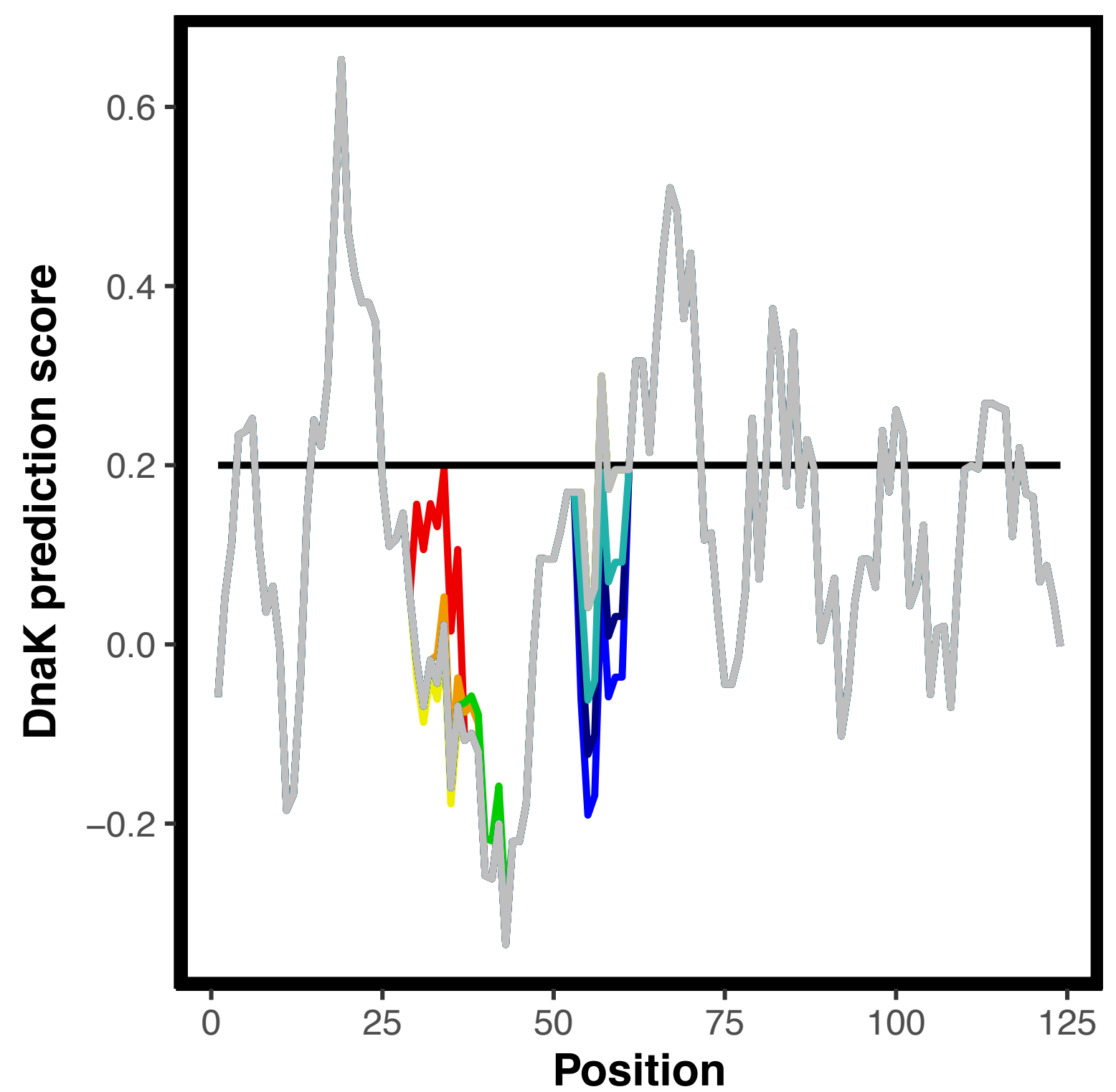

S 1 Prediction of DnaK binding sites in N102LT variants. DnaK binding sites predicted by the qualitative ChaperISM algorithm \{Guiterres, 2020\} for variants: L57G (dark blue), L57C (blue), L57P (cyan), M40A (green), Q33S (yellow), V36I (orange), Q33Y (red) and wt (grey). Where only grey is visible the score is identical for all variants. Cut-off for DnaK binding is 0.2 (black line). 


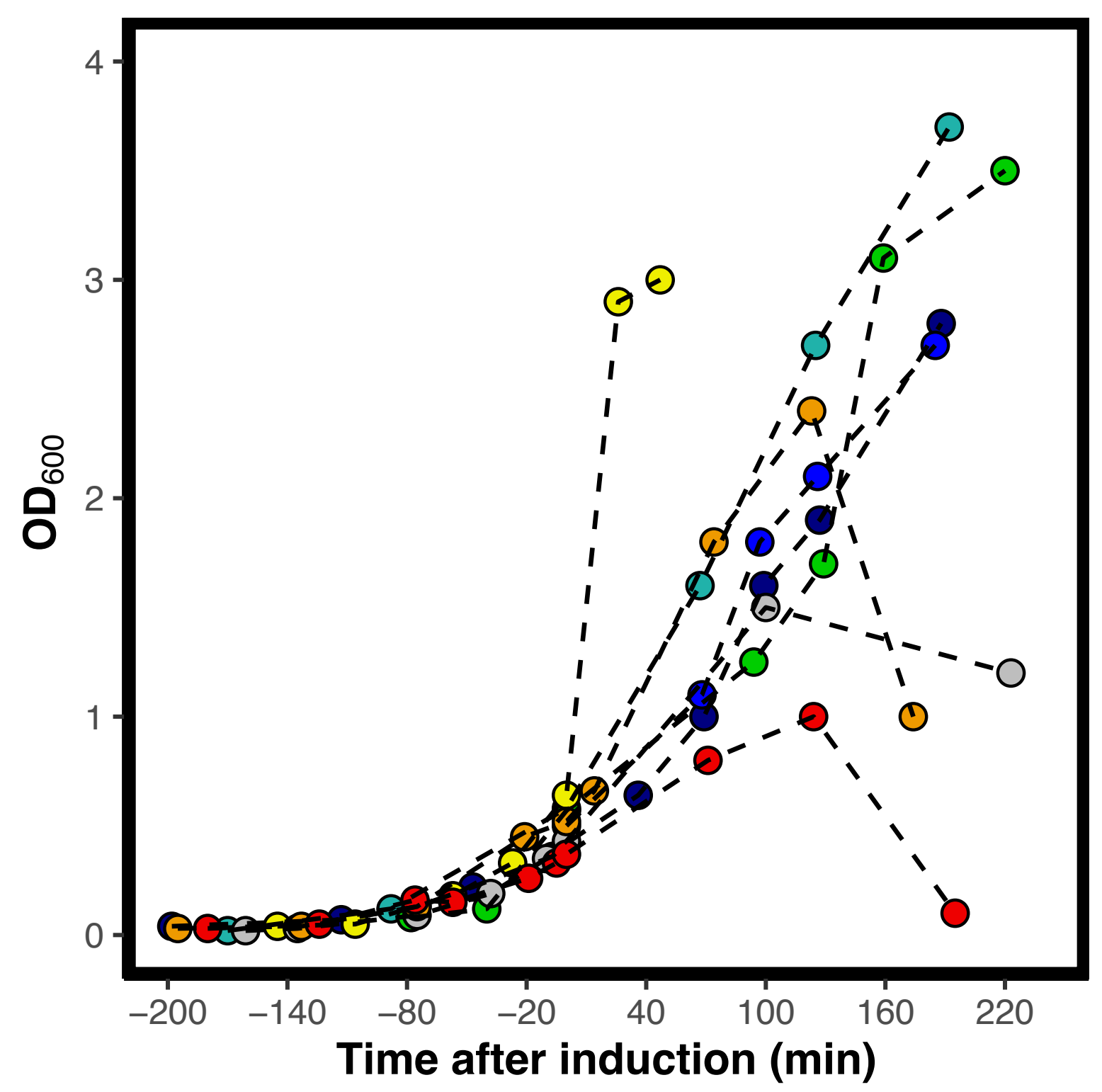

S 2 Growth of cells expressing N102LT variants. OD 600 as a function of time after induction of protein synthesis for cells expressing the variants: L57G (dark blue), L57C (blue), L57P (cyan), M40A (green), Q33S (yellow), V36I (orange), Q33Y (red) and wt (grey). One of two replicates are shown for each variant. 


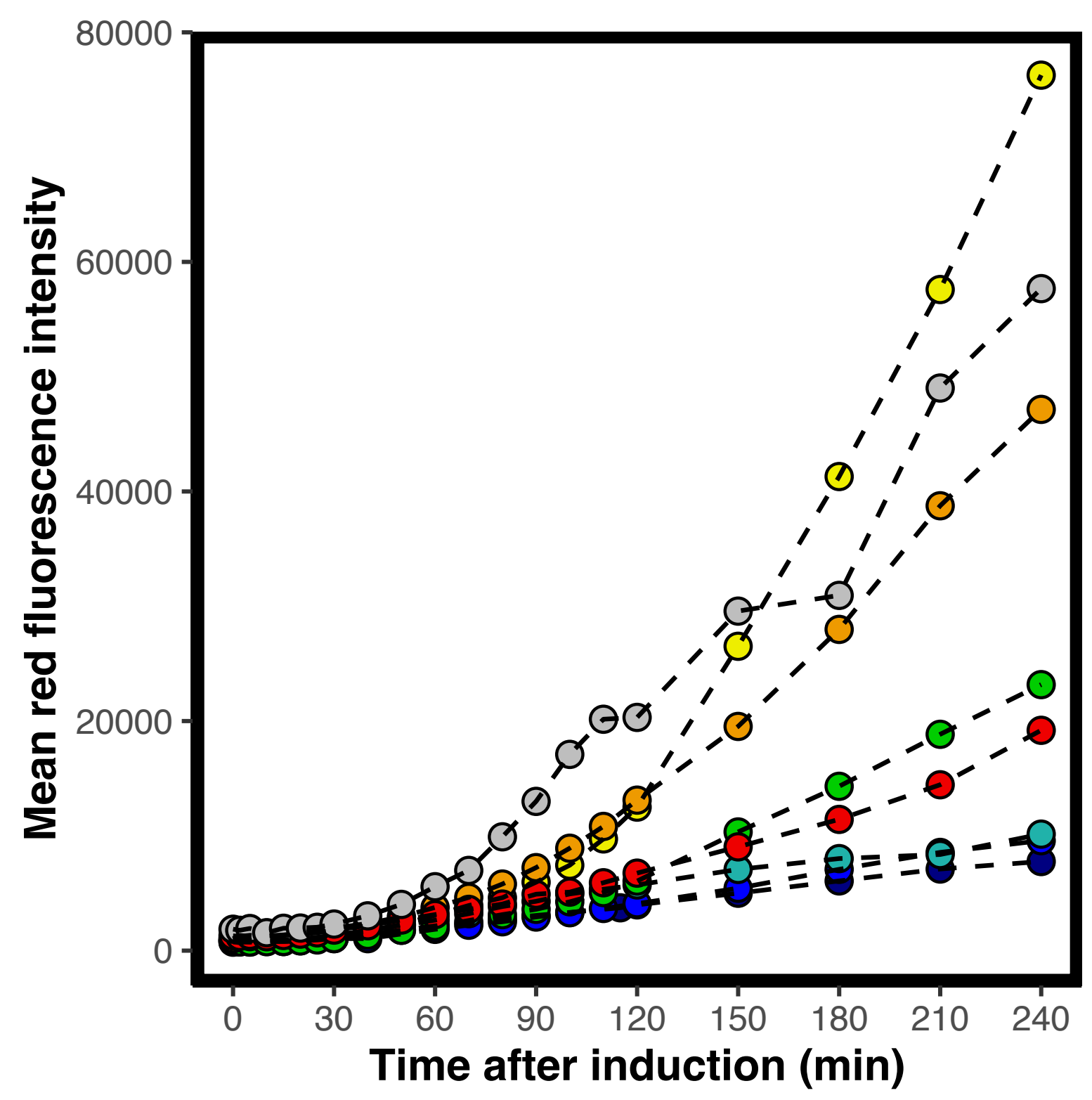

S 3 Abundance of tagRFP fusion proteins monitored by red fluorescence. Mean red fluorescence intensity of cells expressing variants: L57G (dark blue), L57C (blue), L57P (cyan), M40A (green), Q33S (yellow), V36I (orange), Q33Y (red) and wt (grey). One of two replicates are shown for each variant. 


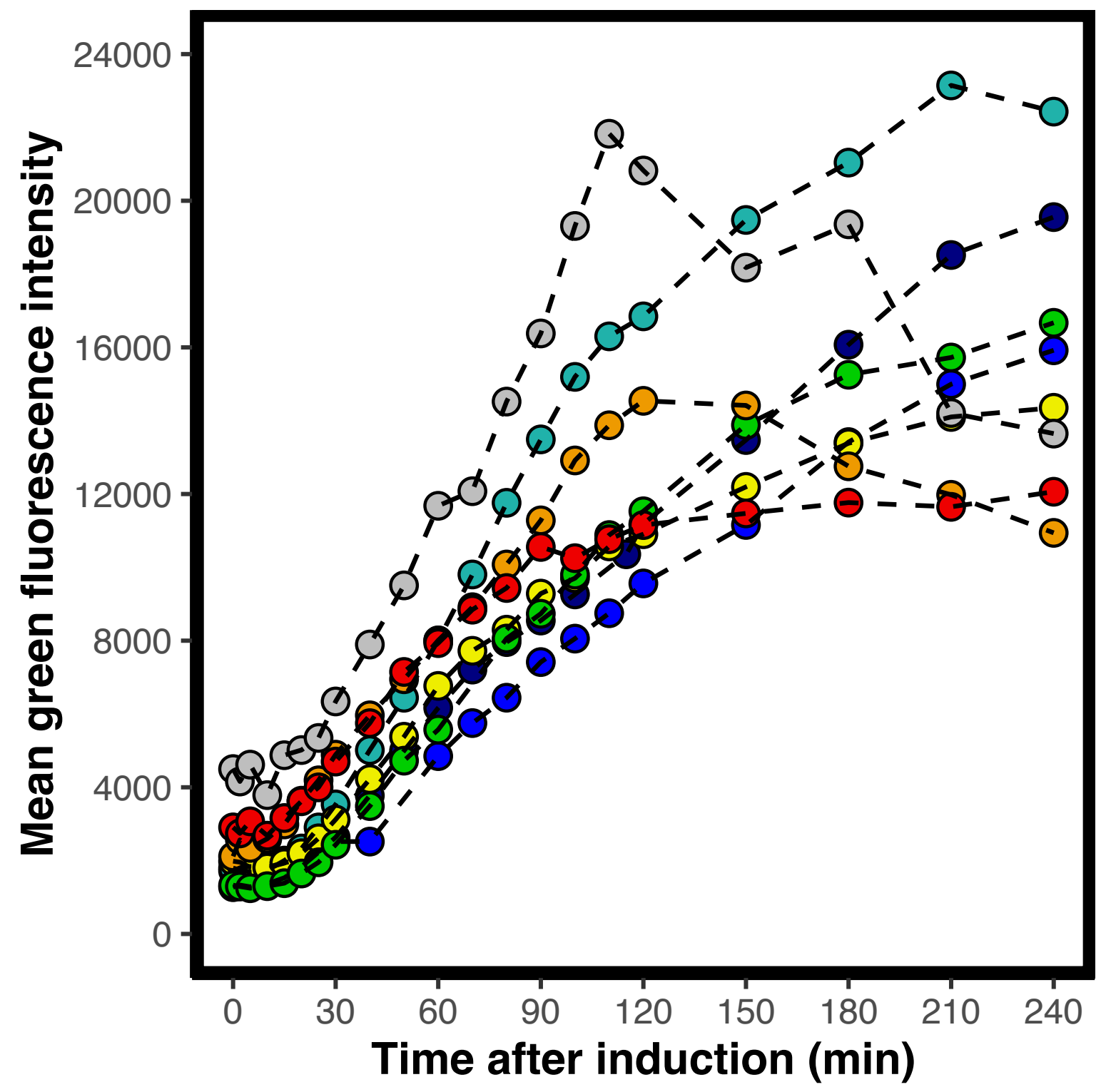

S 4 Transcription from the DnaK promoter monitored by green fluorescence. Mean green fluorescence intensity of cells expressing variants: L57G (dark blue), L57C (blue), L57P (cyan), M40A (green), Q33S (yellow), V36I (orange), Q33Y (red) and wt (grey). One of two replicates are shown for each variant. 


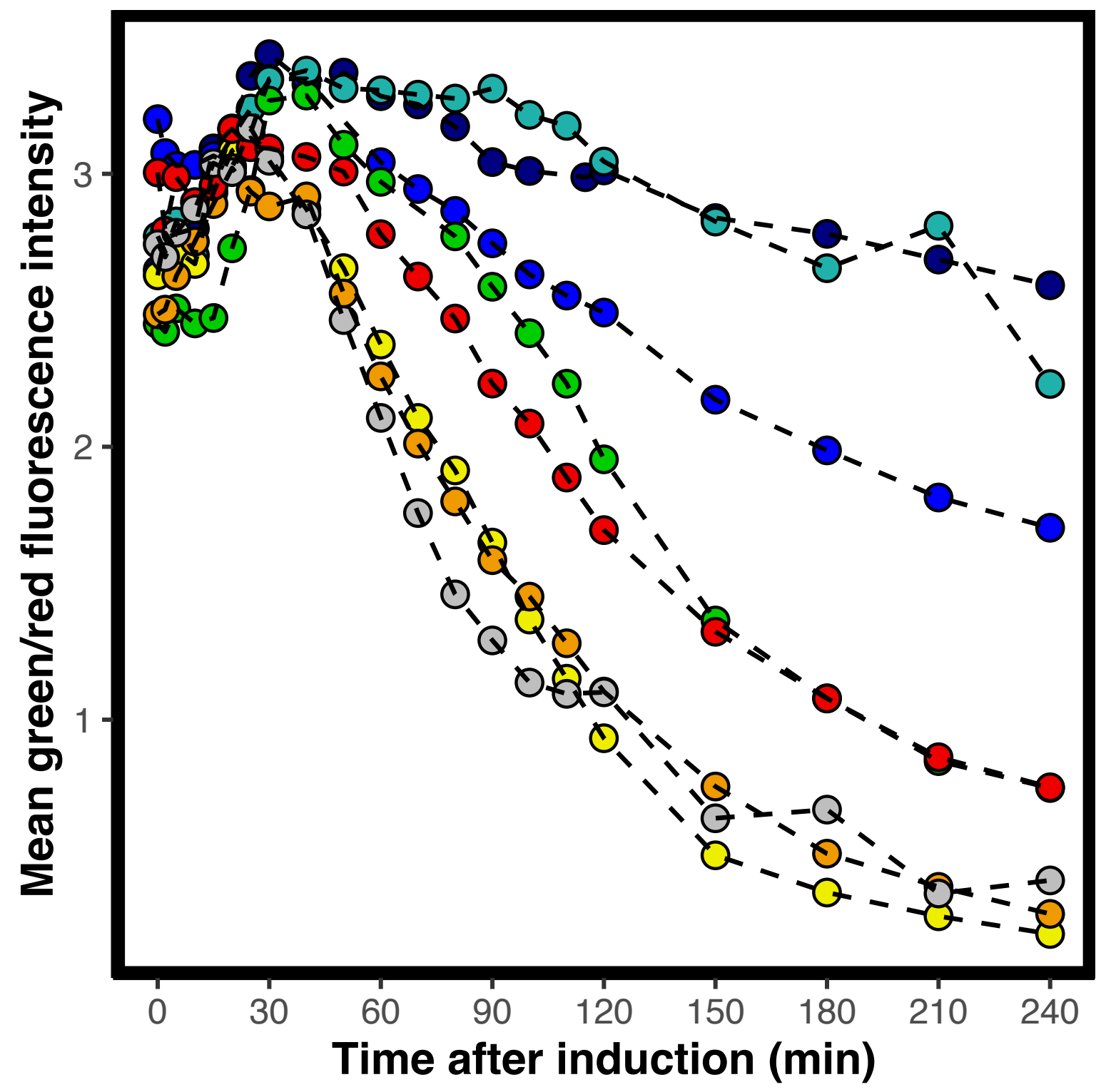

S 5 Accumulated transcription from the DnaK promoter normalised to protein abundance Mean green fluorescence intensity divided by red fluorescence intensity of cells expressing variants: L57G (dark blue), L57C (blue), L57P (cyan), M40A (green), Q33S (yellow), V36I (orange), Q33Y (red) and wt (grey). One of two replicates are shown for each variant. 

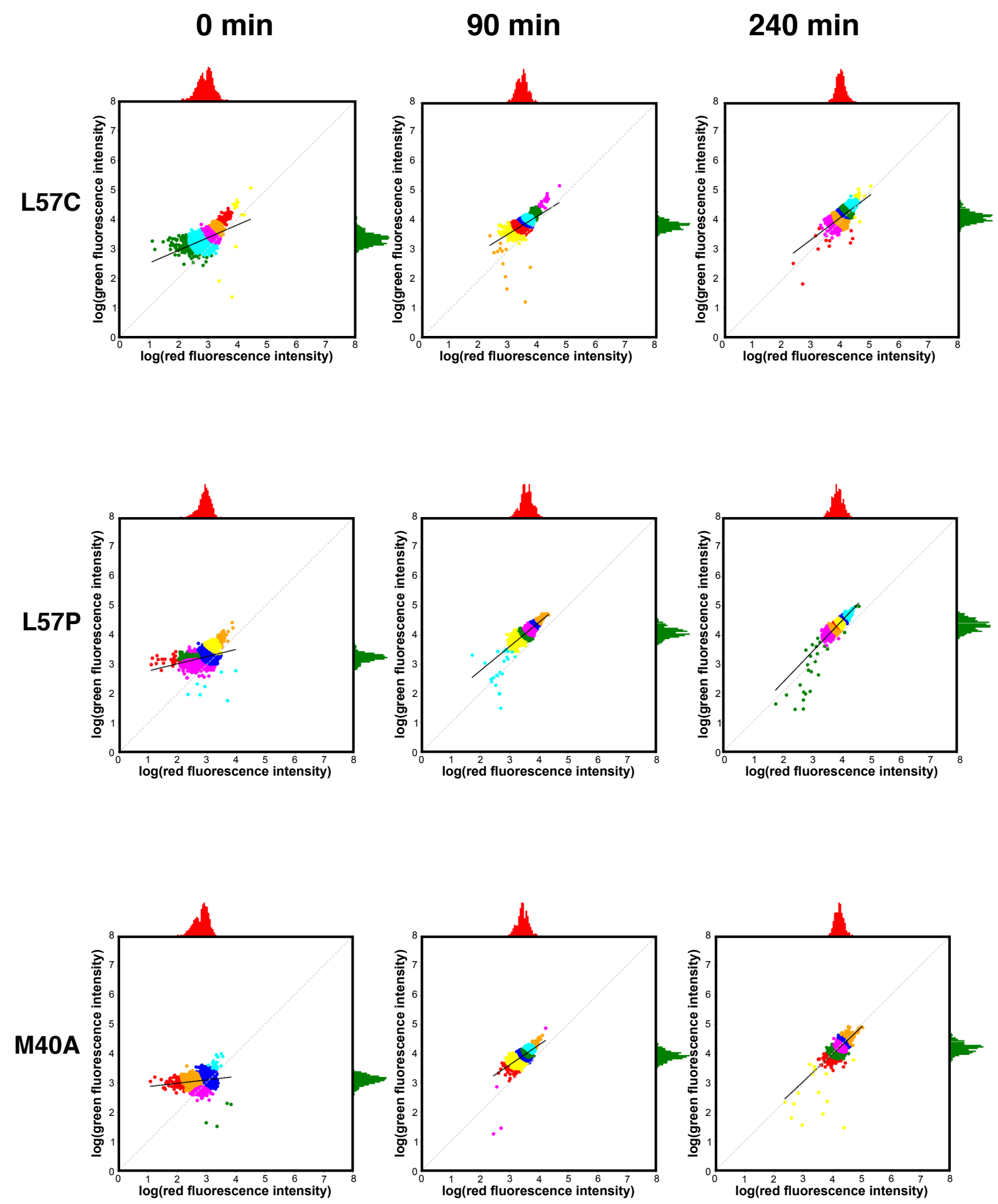

S 6 Red and green fluorescence of single cells expressing L57C, L57P and M40A.The red and green fluorescence for each individual cell in a population expressing L57C (top), L57P (middle) and M40A (bottom) at the time of induction (left) and 240 min after induction with IPTG (right). Colouring is based on a Bayesian Gaussian mixture model for cluster assignment. The x-axis histogram (red) shows the distribution of red fluorescence, and the y-axis histogram (green) shows the distribution of green fluorescence within the population. 


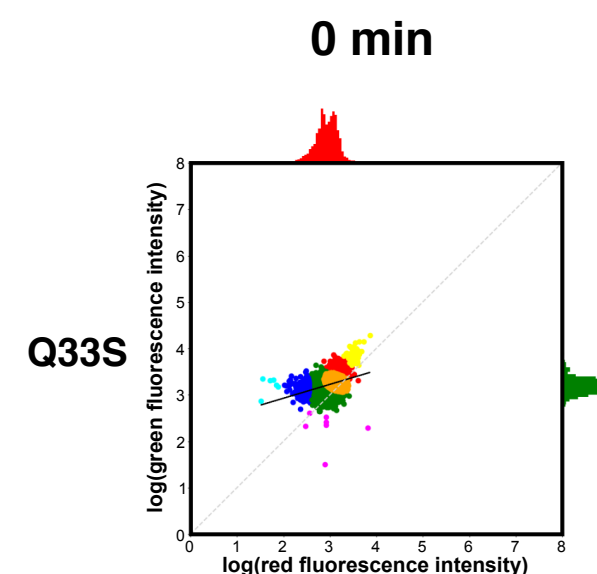

$\log \left(\begin{array}{c}2 \\ \text { (red fluorescence intensity) }\end{array}\right.$
$90 \mathrm{~min}$

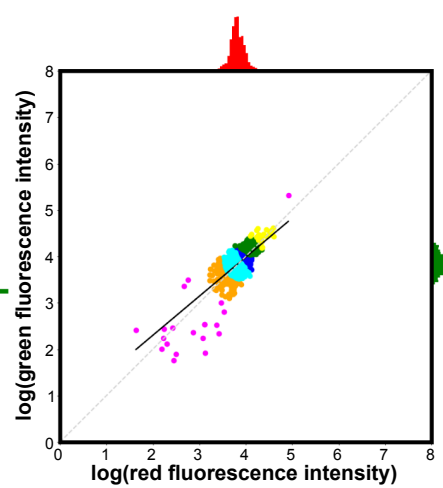

$240 \min$

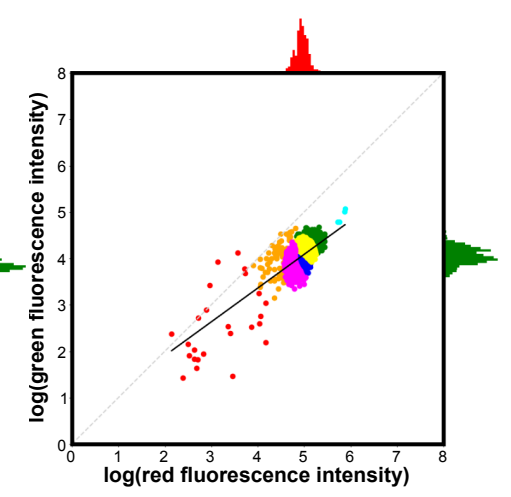

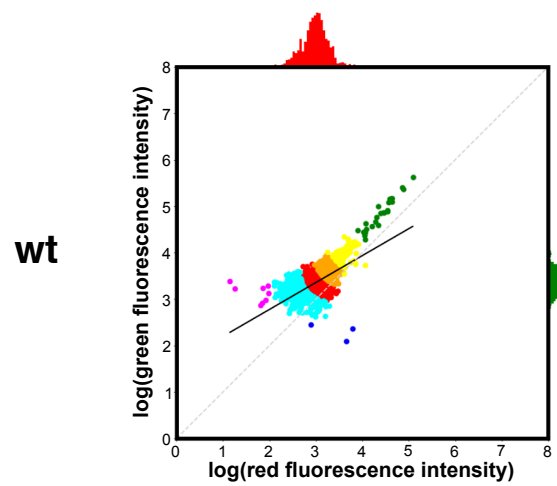
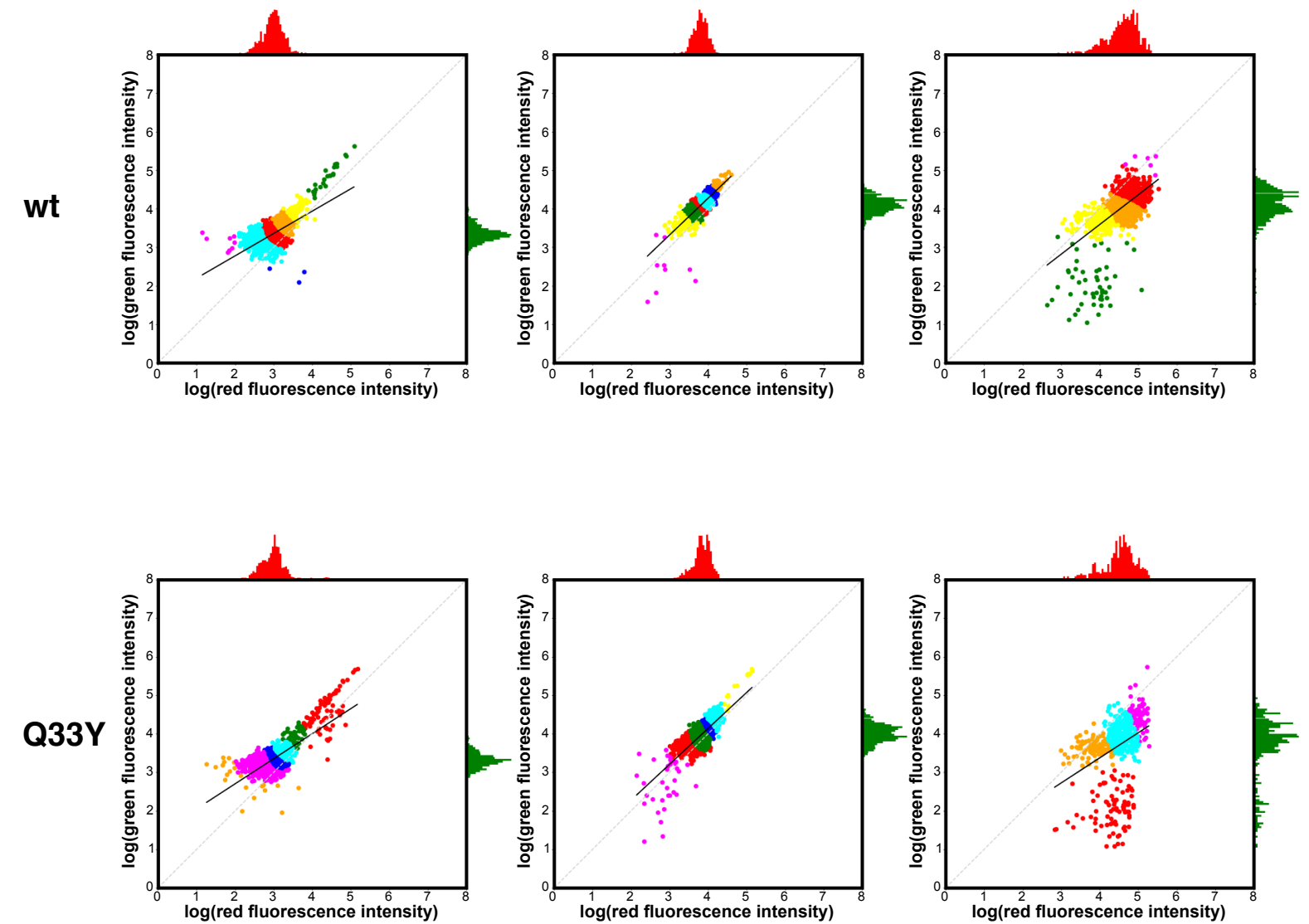

S 7 Red and green fluorescence of single cells expressing Q33S, wt and Q33Y. The red and green fluorescence for each individual cell in a population expressing Q33S (top), wt (middle) and Q33Y (bottom) at the time of induction (left) and 240 min after induction with IPTG (right). Colouring is based on a Bayesian Gaussian mixture model for cluster assignment. The x-axis histogram (red) shows the distribution of red fluorescence, and the y-axis histogram (green) shows the distribution of green fluorescence within the population. 


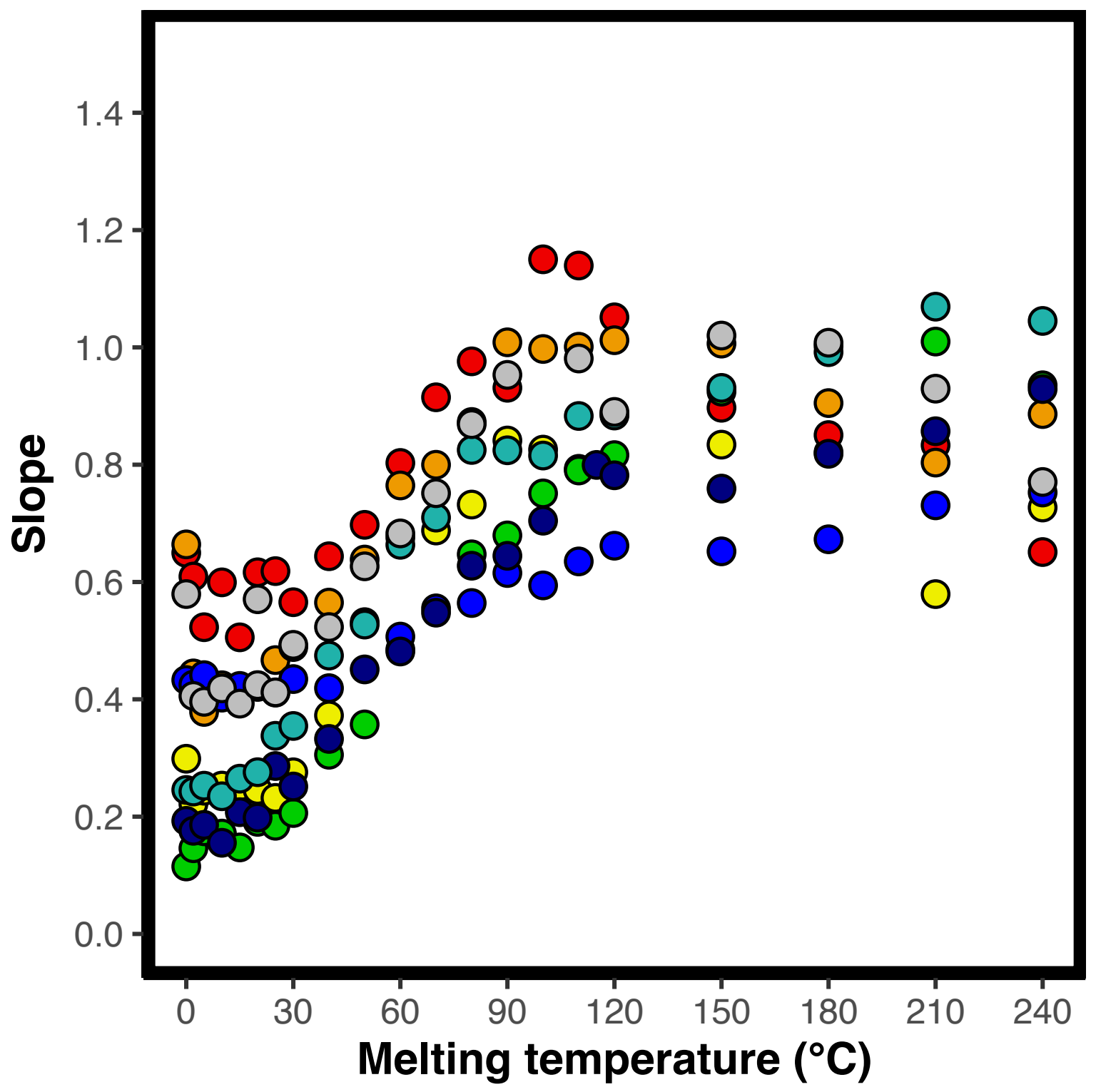

S 8 Slope of fluorescent population over time. For a population of cells expressing the variants: L57G (dark blue), L57C (blue), L57P (cyan), M40A (green), Q33S (yellow), V36I (orange), Q33Y (red) and wt (grey) the slope of log transformed red fluorescence vs log transformed green fluorescence is calculated. One of two replicates are shown for each variant. 


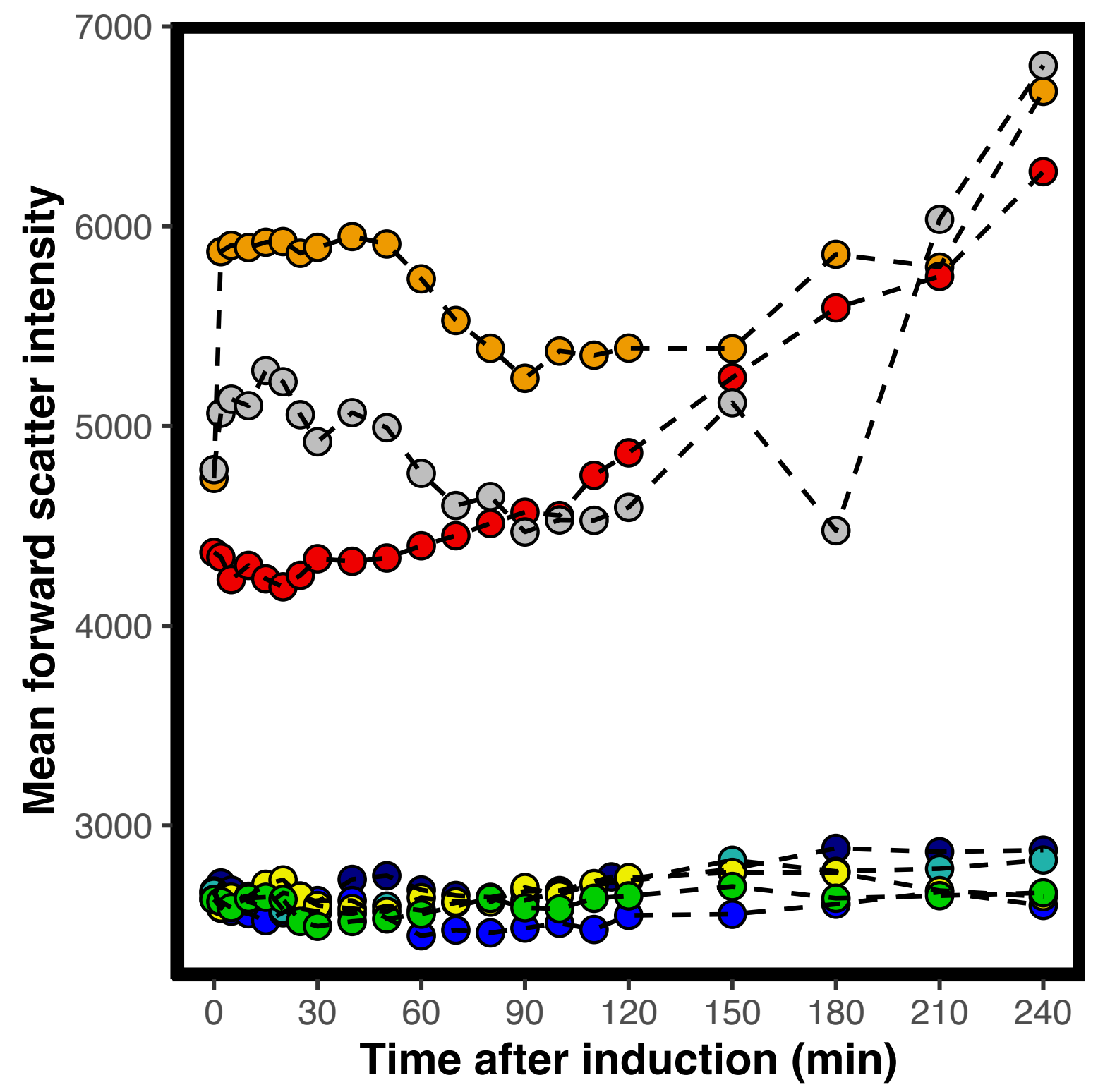

S 9 Forward scatter of whole populations expressing N102LT variants. Forward scatter as a function of time for variants: L57G (dark blue), L57C (blue), L57P (cyan), M40A (green), Q33S (yellow), V36I (orange), Q33Y (red) and wt (grey) One of two replicates are shown for each variant. 


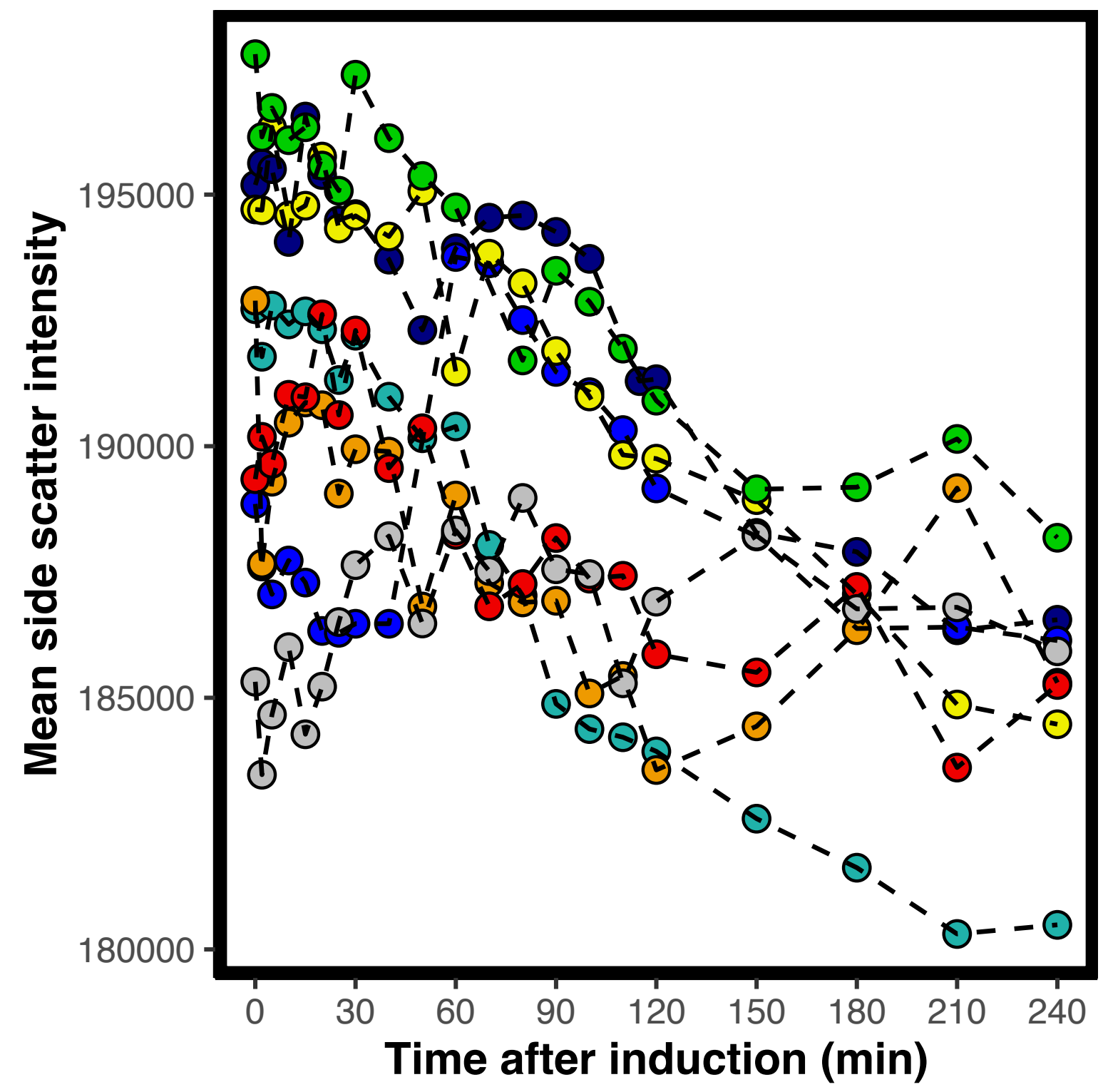

S 10 Side scatter of whole populations expressing N102Lt variants. Side scatter as a function of time for variants: L57G (dark blue), L57C (blue), L57P (cyan), M40A (green), Q33S (yellow), V36I (orange), Q33Y (red) and wt (grey). One of two replicates are shown for each variant. 